\title{
Trace operators of the bi-Laplacian and applications *
}

\author{
Thomas Führer $^{\dagger} \quad$ Alexander Haberl ${ }^{\ddagger} \quad$ Norbert Heuer ${ }^{\dagger}$
}

\begin{abstract}
We study several trace operators and spaces that are related to the bi-Laplacian. They are motivated by the development of ultraweak formulations for the bi-Laplace equation with homogeneous Dirichlet condition, but are also relevant to describe conformity of mixed approximations.

Our aim is to have well-posed (ultraweak) formulations that assume low regularity, under the condition of an $L_{2}$ right-hand side function. We pursue two ways of defining traces and corresponding integration-by-parts formulas. In one case one obtains a non-closed space. This can be fixed by switching to the Kirchhoff-Love traces from [Führer, Heuer, Niemi, An ultraweak formulation of the Kirchhoff-Love plate bending model and DPG approximation, Math. Comp., 88 (2019)]. Using different combinations of trace operators we obtain two well-posed formulations. For both of them we report on numerical experiments with the DPG method and optimal test functions.

In this paper we consider two and three space dimensions. However, with the exception of a given counterexample in an appendix (related to the non-closedness of a trace space), our analysis applies to any space dimension larger than or equal to two.
\end{abstract}

Key words: bi-Laplacian, biharmonic operator, trace operator, fourth-order elliptic PDE, ultraweak formulation, discontinuous Petrov-Galerkin method, optimal test functions AMS Subject Classification: 35J35, 74K20, 74S05, 65N30, 35J67

\section{Introduction}

The bi-Laplace operator and biharmonic functions have generated sustained interest in the mathematics community until today. Just in numerical analysis, MathSciNet reports well beyond 500 publications with these key words in their titles. An early overview of numerical methods for the Dirichlet problem of the bi-Laplacian is given by Glowinski and Pironneau in [16]. A more recent discussion can be found in the introduction of [5].

Our interest in this operator arose while studying the Kirchhoff-Love plate bending model and its numerical approximation by the discontinuous Petrov-Galerkin method with optimal

\footnotetext{
*Supported by CONICYT through FONDECYT projects 11170050, 1190009.

${ }^{\dagger}$ Facultad de Matemáticas, Pontificia Universidad Católica de Chile, Avenida Vicuña Mackenna 4860, Santiago, Chile, email: \{tofuhrer, nheuer\}@mat.uc.cl

${ }^{\ddagger}$ Institute for Analysis and Scientific Computing, Technische Universität Wien, Austria, email: alexander.haberl@asc.tuwien.ac.at
} 
test functions (DPG method). It is well known that the Kirchhoff-Love model (with constant coefficients) reduces to the bi-Laplace equation when considering the deflection of the plate as the only unknown.

In this paper we introduce and analyze trace operators that stem from the bi-Laplacian and relate to integration-by-parts formulas. Such operators are of general interest as they characterize interface conditions for (piecewise) sufficiently smooth functions to be globally in the domain of the bi-Laplacian, or the subordinated Laplacian when considering the Laplacian of the unknown as independent unknown. Specifically, this analysis is required to construct conforming finite element spaces of minimal regularity. Regularity is a delicate issue when splitting the bi-Laplace equation into two Laplace equations (explicitly, or implicitly through a mixed formulation). Early papers on this technique are by Ciarlet and Raviart [4], and Monk [20]. Regularity issues at corners have been analyzed, e.g., in [14, 7]. Thus, our aim is to use the least possible regularity subject to a given right-hand side function in $L_{2}$. We note that Zulehner [21] presents a formulation (and space) where less regular right-hand side functions are permitted.

We consider Dirichlet boundary conditions, that is, a clamped plate in the two-dimensional case. Here we only note that, in principle, it is possible to study different boundary conditions, but the regularity of solutions will depend on them and some technical details can be tricky.

In the rest of this paper we motivate our definitions and analysis by requirements for the DPG method. For instance, the right-hand side function to be in $L_{2}$ is such a requirement. Considering this method, there are good reasons to use ultraweak variational formulations. From the mathematical point of view they simplify the analysis of well-posedness as they allow for exact representations of adjoint operators, cf. [8]. From a practical point of view they give access to approximations of field variables that are close to optimal in the $L_{2}$ sense, cf., e.g., [10, 18, for singularly perturbed problems. For general second order elliptic problems, the $L_{2}$-optimality up to higher order terms is proved in [11. Now, since field variables of ultraweak formulations are only $L_{2}$-elements, the inherent regularity of the underlying problem is passed onto appearing traces. Therefore, the study of trace spaces is at the heart of proving well-posedness of ultraweak formulations. As explained before, the appearing traces (and trace spaces) are equally relevant for the underlying problem and other variational formulations as they precisely describe the notion of conformity and represent tools for its study.

It is the nature of DPG methods to use product test spaces (defined on meshes). This is a fundamental paradigm proposed by Demkowicz and Gopalakrishnan in [9]. For that reason, our traces will live in product spaces related to the boundaries of elements. Nevertheless, our results will apply to operations on domains without mesh, simply by using meshes that consist of a single element.

The remainder of this paper is as follows. In the next section we fix our model problem and introduce a setting needed to develop ultraweak variational formulations. This approach motivates the framework in which we study trace and jump operators, and trace spaces, and is presented in $\$ 3$. Aiming at lowest regularity, we first develop a setting where the unknown $u$ of the bi-Laplace equation and its Laplacian (as independent unknown) are considered as elements of the same regularity $\left(L_{2}\right.$-functions whose Laplacian is in $\left.L_{2}\right)$. This is done in $\$ 3.2$. Later, in \$5. we present a variational formulation based on this framework, state its well-posedness and 
equivalence with the model problem (Theorem 11), and prove the quasi-optimal convergence of the induced DPG scheme (Theorem 12). For this formulation, discrete subspaces with good approximation properties seem to require coupled basis functions (trace components are not independent). This limits the practicality of the induced DPG scheme. We therefore also consider the option of using more regular test functions (then trace components can be approximated separately). This change gives rise to different trace operators ( $\operatorname{tr}^{\Delta, 2}$ acting on $u$, and $\operatorname{tr}^{2, \Delta}$ acting on $\sigma=\Delta u$ ) and spaces. They are studied in $\$ 3.3$. Unfortunately, it turns out that the image of $\operatorname{tr}^{2, \Delta}$ is not closed (this is proved in Appendix A). We therefore embed this space in a larger, closed trace space known from our Kirchhoff-Love traces studied in [13]. The corresponding variational formulation and DPG scheme are presented in \$5, stating well-posedness and quasioptimal convergence by Theorems 13 and 14, respectively. Proofs of Theorems 11 and 13 are given in 8 . We do not provide a discrete analysis here, but we do present some numerical experiments in $\$ 7$. They illustrate expected convergence properties.

One conclusion of our analysis is that the solution $u$ to the bi-Laplace equation with righthand side function in $L_{2}$ and homogeneous boundary condition satisfies $u \in H^{2}$. Since we have not seen this result in the literature, we resume and prove this statement in Appendix C. There are, however, related $H^{2}$-regularity results for the bi-Laplacian by Girault and Raviart in [15, $\S 5]$ (dimensions 2 and 3), and by De Coster et al. [7] for corner-type domains in $\mathbb{R}^{2}$.

Throughout the paper, $a \lesssim b$ means that $a \leq c b$ with a generic constant $c>0$ that is independent of the underlying mesh (except for possible general restrictions like shape-regularity of elements). Similarly, we use the notation $a \simeq b$.

\section{Model problem}

Let $\Omega \subset \mathbb{R}^{d}(d \in\{2,3\})$ be a bounded simply connected Lipschitz domain. (We remark that our analysis and results will apply to any space dimension $d \geq 2$, with the exception of Lemma 10 with respect to the Dirac distributions and the counterexample of Appendix A. Nevertheless, we restrict ourselves to $d \in\{2,3\}$ since we will make use of some results from [13] which are true in any space dimension $d \geq 2$, but are only formulated for $d \in\{2,3\}$.)

The boundary of $\Omega$ is denoted by $\Gamma=\partial \Omega$ with exterior unit normal vector $\mathbf{n}$. For given $f \in L_{2}(\Omega)$ our model problem is

$$
\begin{array}{rlr}
\Delta^{2} u=f & \text { in } \Omega, \\
u=\partial_{\mathbf{n}} u=0 & \text { on } \Gamma .
\end{array}
$$

We intend to develop an ultraweak formulation of (1) with product test spaces. To this end we consider a mesh $\mathcal{T}$ that consists of general non-intersecting Lipschitz elements. To the mesh $\mathcal{T}=\{T\}$ we assign the skeleton $\mathcal{S}=\{\partial T ; T \in \mathcal{T}\}$.

Introducing $\sigma:=\Delta u$, we test the two equations $\Delta \sigma=f, \Delta u-\sigma=0$ on any $T \in \mathcal{T}$ by sufficiently smooth functions $v$ and $\tau$, respectively, and integrate by parts twice. This formally gives

$$
(\sigma, \Delta v)_{T}+\left\langle\partial_{\mathbf{n}} \sigma, v\right\rangle_{\partial T}-\left\langle\sigma, \partial_{\mathbf{n}} v\right\rangle_{\partial T}+(u, \Delta \tau)_{T}+\left\langle\partial_{\mathbf{n}} u, \tau\right\rangle_{\partial T}-\left\langle u, \partial_{\mathbf{n}} \tau\right\rangle_{\partial T}-(\sigma, \tau)_{T}=(f, v)_{T},
$$


where $(\cdot, \cdot)_{T}$ denotes the $L_{2}(T)$-duality. We still have to interpret the dualities on $\partial T$ denoted by $\langle\cdot, \cdot\rangle_{\partial T}$. Summing over $T \in \mathcal{T}$, we obtain, again formally,

$$
\begin{aligned}
& (u, \Delta \tau)_{\mathcal{T}}+(\sigma, \Delta v-\tau)_{\mathcal{T}} \\
& +\sum_{T \in \mathcal{T}}\left\langle\partial_{\mathbf{n}} \sigma, v\right\rangle_{\partial T}-\sum_{T \in \mathcal{T}}\left\langle\sigma, \partial_{\mathbf{n}} v\right\rangle_{\partial T}+\sum_{T \in \mathcal{T}}\left\langle\partial_{\mathbf{n}} u, \tau\right\rangle_{\partial T}-\sum_{T \in \mathcal{T}}\left\langle u, \partial_{\mathbf{n}} \tau\right\rangle_{\partial T}=(f, v) .
\end{aligned}
$$

Here and in the following, $(\cdot, \cdot)_{\mathcal{T}}$ denotes the $L_{2}$-duality in the product space $L_{2}(\mathcal{T})$, meaning that appearing differential operators are taken piecewise with respect to $T \in \mathcal{T}$. Below, we also use the notation of differential operators with index $\mathcal{T}$ to indicate piecewise operations, e.g., $\left(\Delta_{\mathcal{T}} u, v\right)=(\Delta u, v)_{\mathcal{T}}$. Furthermore, from now on, $\mathbf{n}$ denotes a generic unit normal vector on $\partial T$ $(T \in \mathcal{T})$ and $\Gamma$, pointing outside $T$ and $\Omega$, respectively.

Before returning to our formulation (2) we need to study trace operators to give a meaning to the skeleton dualities appearing in (2). This will be done next, before returning to (2) in $\$ 4$. and again in $\$ 5$.

\section{Traces and jumps}

\subsection{Spaces and norms}

Given $T \in \mathcal{T}$, and sufficiently smooth scalar (respectively, symmetric tensor) function $z: T \rightarrow \mathbb{R}$ (respectively, $\Theta: T \rightarrow \mathbb{R}^{d \times d}$ ), we define the norms $\|\cdot\|_{\Delta, T},\|\cdot\|_{2, T}$ and $\|\cdot\|_{\mathrm{dDiv}, T}$ by

$$
\|z\|_{\Delta, T}^{2}:=\|z\|_{T}^{2}+\|\Delta z\|_{T}^{2}, \quad\|z\|_{2, T}^{2}:=\|z\|_{T}^{2}+\|\varepsilon \nabla z\|_{T}^{2}, \quad\|\boldsymbol{\Theta}\|_{\mathrm{dDiv}, T}^{2}:=\|\boldsymbol{\Theta}\|_{T}^{2}+\|\operatorname{div} \operatorname{div} \boldsymbol{\Theta}\|_{T}^{2} .
$$

Here, $\|\cdot\|_{T}$ is the $L_{2}(T)$-norm (for scalar and tensor-valued functions), $\varepsilon(\cdot):=\frac{1}{2}\left(\nabla(\cdot)+\nabla(\cdot)^{\top}\right.$ ) denotes the symmetric gradient, that is, $\varepsilon \nabla z$ is the Hessian of $z$, div is the standard divergence operator, and div is the divergence applied row-wise to tensors. Analogously, we use the corresponding norms on $\Omega$ where we drop the index $T$. For instance, $\|\cdot\|$ is the $L_{2}(\Omega)$-norm. We also need the $L_{2}(\Omega)$-bilinear form $(\cdot, \cdot)$, for scalar and tensor functions.

We define the spaces $H(\Delta, T)$ and $H^{2}(T)$ as the closures of $\mathcal{D}(\bar{T})$ with respect to the norms $\|\cdot\|_{\Delta, T}$ and $\|\cdot\|_{2, T}$, respectively. Correspondingly, $H(\operatorname{div} \operatorname{div}, T)$ is the closure of the space of smooth symmetric tensors on $T$ with respect to $\|\cdot\|_{\mathrm{dDiv}, T}$. Analogously, $H(\Delta, \Omega)$ and $H_{0}^{2}(\Omega)$ are the respective closures of $\mathcal{D}(\bar{\Omega})$ and $\mathcal{D}(\Omega)$, with norms $\|\cdot\|_{\Delta}$ and $\|\cdot\|_{2}$, and $H(\operatorname{div} \operatorname{div}, \Omega)$ is the closure with respect to $\|\cdot\|_{\mathrm{d} \text { Div }}$ of the space of smooth symmetric tensors on $\Omega$.

Given the mesh $\mathcal{T}$, we will need the induced product spaces

$$
\begin{aligned}
H(\Delta, \mathcal{T}) & :=\left\{z \in L_{2}(\Omega) ;\left.z\right|_{T} \in H(\Delta, T) \forall T \in \mathcal{T}\right\} \\
H^{2}(\mathcal{T}) & :=\left\{z \in L_{2}(\Omega) ;\left.z\right|_{T} \in H^{2}(T) \forall T \in \mathcal{T}\right\} \\
H(\operatorname{div} \operatorname{div}, \mathcal{T}) & :=\left\{\boldsymbol{\Theta} \in \mathbb{L}_{2}^{s}(\Omega) ;\left.\boldsymbol{\Theta}\right|_{T} \in H(\operatorname{div} \operatorname{div}, T) \forall T \in \mathcal{T}\right\}
\end{aligned}
$$

with canonical product norms $\|\cdot\|_{\Delta, \mathcal{T}},\|\cdot\|_{2, \mathcal{T}}$, and $\|\cdot\|_{\mathrm{dDiv}}, \mathcal{T}$, respectively. Here, $\mathbb{L}_{2}^{s}$ indicates the space of symmetric $L_{2}$-tensors on the indicated domain. 


\subsection{Traces and jumps, part one}

We define linear operators $\operatorname{tr}_{T}^{\Delta}: H(\Delta, T) \rightarrow H(\Delta, T)^{\prime}$ for $T \in \mathcal{T}$ by

$$
\left\langle\operatorname{tr}_{T}^{\Delta}(z), v\right\rangle_{\partial T}:=(\Delta v, z)_{T}-(v, \Delta z)_{T} \quad \forall v \in H(\Delta, T)
$$

and observe that, for sufficiently smooth functions $v$ and $z$,

$$
\left\langle\operatorname{tr}_{T}^{\Delta}(z), v\right\rangle_{\partial T}=\left\langle z, \partial_{\mathbf{n}} v\right\rangle_{\partial T}-\left\langle v, \partial_{\mathbf{n}} z\right\rangle_{\partial T}
$$

with $L_{2}(\partial T)$-duality $\langle\cdot, \cdot\rangle_{\partial T}$ and standard trace and normal derivative. In other words, the trace operator $\operatorname{tr}_{T}^{\Delta}$ can deliver standard traces (trace and normal derivative) on $\partial T$ when diverting from the setting as a map from $H(\Delta, T)$ to its dual. This will be further discussed in 3.3 below. Note the duality

$$
\left\langle\operatorname{tr}_{T}^{\Delta}(z), v\right\rangle_{\partial T}=-\left\langle\operatorname{tr}_{T}^{\Delta}(v), z\right\rangle_{\partial T} \quad \forall z, v \in H(\Delta, T) .
$$

The range of $\operatorname{tr}_{T}^{\Delta}$ is

$$
\mathbf{H}^{\Delta}(\partial T):=\operatorname{tr}_{T}^{\Delta}(H(\Delta, T) \quad(T \in \mathcal{T})
$$

Switching from individual elements $T \in \mathcal{T}$ to the whole of $\mathcal{T}$, a collective trace operator is defined by

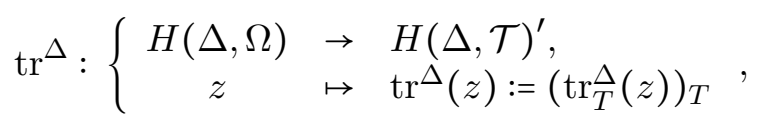

with duality

$$
\left\langle\operatorname{tr}^{\Delta}(z), v\right\rangle_{\mathcal{S}}:=\sum_{T \in \mathcal{T}}\left\langle\operatorname{tr}_{T}^{\Delta}(z), v\right\rangle_{\partial T} \quad(z \in H(\Delta, \Omega), v \in H(\Delta, \mathcal{T}))
$$

To define a trace space that reflects the homogeneous boundary condition under consideration, we make use of the operator $\operatorname{tr}_{\Omega}^{\Delta}$ that is defined like $\operatorname{tr}_{T}^{\Delta}$ by replacing $T$ with $\Omega$ :

$$
\left\langle\operatorname{tr}_{\Omega}^{\Delta}(z), v\right\rangle_{\Gamma}:=(z, \Delta v)-(\Delta z, v) \quad(z, v \in H(\Delta, \Omega)) .
$$

Then, with

$$
H_{0}(\Delta, \Omega):=\operatorname{ker}\left(\operatorname{tr}_{\Omega}^{\Delta}\right)
$$

we introduce the product trace spaces

$$
\mathbf{H}_{00}^{\Delta}(\mathcal{S}):=\operatorname{tr}^{\Delta}\left(H_{0}(\Delta, \Omega)\right) \subset \mathbf{H}^{\Delta}(\mathcal{S}):=\operatorname{tr}^{\Delta}(H(\Delta, \Omega)) \subset H(\Delta, \mathcal{T})^{\prime} .
$$

Below, we refer to elements of such skeleton trace spaces in the form, e.g., $\widehat{\boldsymbol{v}}=\left(\widehat{\boldsymbol{v}}_{T}\right)_{T \in \mathcal{T}}$.

The local and global trace spaces are equipped with the canonical trace norms,

$$
\begin{array}{ll}
\|\widehat{\boldsymbol{v}}\|_{\Delta, \partial T}=\inf \left\{\|v\|_{\Delta, T} ; v \in H(\Delta, T), \operatorname{tr}_{T}^{\Delta}(v)=\widehat{\boldsymbol{v}}\right\} & \left(\widehat{\boldsymbol{v}} \in \mathbf{H}^{\Delta}(\partial T), T \in \mathcal{T}\right), \\
\|\widehat{\boldsymbol{v}}\|_{\Delta, \mathcal{S}}=\inf \left\{\|v\|_{\Delta} ; v \in H(\Delta, \Omega), \operatorname{tr}^{\Delta}(v)=\widehat{\boldsymbol{v}}\right\} & \left(\widehat{\boldsymbol{v}} \in \mathbf{H}^{\Delta}(\mathcal{S}) \cup \mathbf{H}_{00}^{\Delta}(\mathcal{S})\right) .
\end{array}
$$


(Obviously, $\mathbf{H}_{00}^{\Delta}(\mathcal{S})$ is a subspace of $\mathbf{H}^{\Delta}(\mathcal{S})$. But here, and in some instances below, we write $\mathbf{H}^{\Delta}(\mathcal{S}) \cup \mathbf{H}_{00}^{\Delta}(\mathcal{S})$ to stress the fact that both spaces are furnished with the same norm.) Alternative norms are defined by duality,

$$
\begin{aligned}
\|\widehat{\boldsymbol{v}}\|_{\Delta^{\prime}, \partial T} & :=\sup _{0 \neq z \in H(\Delta, T)} \frac{\langle\widehat{\boldsymbol{v}}, z\rangle_{\partial T}}{\|z\|_{\Delta, T}} \quad\left(\widehat{\boldsymbol{v}} \in \mathbf{H}^{\Delta}(\partial T), T \in \mathcal{T}\right), \\
\|\widehat{\boldsymbol{v}}\|_{\Delta^{\prime}, \mathcal{S}} & :=\sup _{0 \neq z \in H(\Delta, \mathcal{T})} \frac{\langle\widehat{\boldsymbol{v}}, z\rangle_{\mathcal{S}}}{\|z\|_{\Delta, \mathcal{T}}} \quad\left(\widehat{\boldsymbol{v}} \in \mathbf{H}^{\Delta}(\mathcal{S}) \cup \mathbf{H}_{00}^{\Delta}(\mathcal{S})\right) .
\end{aligned}
$$

Here, the dualities on $\partial T$ and $\mathcal{S}$ are given by the corresponding trace operations, (3) for the local spaces and (5) on $\mathcal{S}$. For instance, the duality between $\widehat{\boldsymbol{v}} \in \mathbf{H}^{\Delta}(\partial T)$ and $z \in H(\Delta, T)$ is $\langle\widehat{\boldsymbol{v}}, z\rangle_{\partial T}=(\Delta z, v)_{T}-(z, \Delta v)_{T}$ with arbitrary $v \in H(\Delta, T)$ such that $\operatorname{tr}_{T}^{\Delta}(v)=\widehat{\boldsymbol{v}}$.

Lemma 1. It holds the identity

$$
\|\widehat{\boldsymbol{z}}\|_{\Delta^{\prime}, \partial T}=\|\widehat{\boldsymbol{z}}\|_{\Delta, \partial T} \quad \forall \widehat{\boldsymbol{z}} \in \mathbf{H}^{\Delta}(\partial T), T \in \mathcal{T},
$$

so that

$$
\operatorname{tr}_{T}^{\Delta}: H(\Delta, T) \rightarrow \mathbf{H}^{\Delta}(\partial T)
$$

has unit norm and $\left(\mathbf{H}^{\Delta}(\partial T),\|\cdot\|_{\Delta^{\prime}, \partial T}\right)$ is closed.

Proof. The proof is essentially identical to the one of Lemma 3.2 in [13. We just need to replace spaces, operators and norms by the ones used here. For the convenience of the reader we repeat the proof.

The estimate $\|\widehat{\boldsymbol{z}}\|_{\Delta^{\prime}, \partial T} \leq\|\widehat{\boldsymbol{z}}\|_{\Delta, \partial T}$ is due to the boundedness

$$
\left\langle\operatorname{tr}_{T}^{\Delta}(z), v\right\rangle_{\partial T} \leq\|z\|_{\Delta, T}\|v\|_{\Delta, T} \quad \forall z, v \in H(\Delta, T), T \in \mathcal{T} .
$$

To show the other direction we consider an element $T \in \mathcal{T}$ and $\widehat{\boldsymbol{z}} \in \mathbf{H}^{\Delta}(\partial T)$, and define $v \in$ $H(\Delta, T)$ by solving

$$
(\Delta v, \Delta \delta v)_{T}+(v, \delta v)_{T}=\langle\widehat{\boldsymbol{z}}, \delta v\rangle_{\partial T} \quad \forall \delta v \in H(\Delta, T) .
$$

One deduces that

$$
\Delta^{2} v+v=0 \quad \text { in } L_{2}(T) .
$$

We then define $z \in H(\Delta, T)$ as the solution to

$$
(\Delta z, \Delta \delta z)_{T}+(z, \delta z)_{T}=\left\langle\operatorname{tr}_{T}^{\Delta}(\delta z), v\right\rangle_{\partial T} \quad \forall \delta z \in H(\Delta, T) .
$$

Again, it holds

$$
\Delta^{2} z+z=0 \quad \text { in } L_{2}(T)
$$


Let us show that $z=\Delta v$. To this end we define $z^{*}:=\Delta v$ and find that $\Delta z^{*}=-v$, cf. (8). Using this relation, and the definitions of $z^{*}$ and $\operatorname{tr}_{T}^{\Delta}$, cf. (3), we obtain

$$
\left(\Delta z^{*}, \Delta \delta z\right)_{T}+\left(z^{*}, \delta z\right)_{T}=-(v, \Delta \delta z)_{T}+(\Delta v, \delta z)_{T}=\left\langle\operatorname{tr}_{T}^{\Delta}(\delta z), v\right\rangle_{\partial T}
$$

for any $\delta z \in H(\Delta, T)$. This shows that $z^{*}$ solves (9), that is, $z=z^{*}=\Delta v$. Due to this relation and $\Delta z=-v$, it follows by (7) that

$$
\begin{aligned}
\left\langle\operatorname{tr}_{T}^{\Delta}(z), \delta v\right\rangle_{\partial T} & =(z, \Delta \delta v)_{T}-(\Delta z, \delta v)_{T} \\
& =(\Delta v, \Delta \delta v)_{T}+(v, \delta v)_{T}=\langle\widehat{z}, \delta v\rangle_{\partial T} \quad \forall \delta v \in H(\Delta, T) .
\end{aligned}
$$

In other words, $\operatorname{tr}_{T}^{\Delta}(z)=\widehat{z}$. This relation together with selecting $\delta v=v$ in (7) and $\delta z=z$ in (9), shows that

$$
\langle\widehat{\boldsymbol{z}}, v\rangle_{\partial T}=\|v\|_{\Delta, T}^{2}=\|z\|_{\Delta, T}^{2} .
$$

Noting that $\|z\|_{\Delta, T}=\|\widehat{\boldsymbol{z}}\|_{\Delta, \partial T}$ by $(10)$, this relation finishes the proof of the norm identity. The space $\mathbf{H}^{\Delta}(\partial T)$ is closed as the image of a bounded below operator.

Proposition 2. (i) For $z \in H(\Delta, \mathcal{T})$ it holds

$$
z \in H(\Delta, \Omega) \quad \Leftrightarrow \quad\left\langle\operatorname{tr}^{\Delta}(v), z\right\rangle_{\mathcal{S}}=0 \quad \forall v \in H_{0}(\Delta, \Omega)
$$

and

$$
z \in H_{0}(\Delta, \Omega) \quad \Leftrightarrow \quad\left\langle\operatorname{tr}^{\Delta}(v), z\right\rangle_{\mathcal{S}}=0 \quad \forall v \in H(\Delta, \Omega)
$$

(ii) The identity

$$
\sum_{T \in \mathcal{T}}\left\|\widehat{\boldsymbol{z}}_{T}\right\|_{\Delta, \partial T}^{2}=\|\widehat{\boldsymbol{z}}\|_{\Delta, \mathcal{S}}^{2} \quad \forall \widehat{\boldsymbol{z}}=\left(\widehat{\boldsymbol{z}}_{T}\right)_{T} \in \mathbf{H}^{\Delta}(\mathcal{S}) \cup \mathbf{H}_{00}^{\Delta}(\mathcal{S})
$$

holds true.

Proof. The proof of (i) follows the standard procedure, cf. [1, Proof of Theorem 2.3] and [13, Proof of Proposition 3.8(i)]. For $z \in H(\Delta, \Omega)$ and $v \in H_{0}(\Delta, \Omega)$ we have that

$$
-\left\langle\operatorname{tr}^{\Delta}(z), v\right\rangle_{\mathcal{S}}=\left\langle\operatorname{tr}^{\Delta}(v), z\right\rangle_{\mathcal{S}} \stackrel{\text { def }}{=} \sum_{T \in \mathcal{T}}(\Delta z, v)_{T}-(z, \Delta v)_{T}=(\Delta z, v)-(z, \Delta v)=\left\langle\operatorname{tr}_{\Omega}^{\Delta}(v), z\right\rangle_{\Gamma}=0 .
$$

The penultimate step is due to (6), and the last identity holds $\operatorname{since} \operatorname{tr}_{\Omega}^{\Delta}(v)=0$ by definition of $H_{0}(\Delta, \Omega)$. This is the direction " $\Rightarrow$ " in both statements of part (i).

Now, for given $z \in H(\Delta, \mathcal{T})$ with $\left\langle\operatorname{tr}^{\Delta}(v), z\right\rangle_{\mathcal{S}}=0$ for any $v \in H_{0}(\Delta, \Omega)$ we have in the distributional sense

$$
\Delta z(v)=(z, \Delta v)=(\Delta z, v)_{\mathcal{T}}-\left\langle\operatorname{tr}^{\Delta}(v), z\right\rangle_{\mathcal{S}}=(\Delta z, v)_{\mathcal{T}} \quad \forall v \in \mathcal{D}(\Omega)
$$

Therefore, $\Delta z \in L_{2}(\Omega)$, that is, $z \in H(\Delta, \Omega)$. 
Analogously, if $\left\langle\operatorname{tr}^{\Delta}(v), z\right\rangle_{\mathcal{S}}=0$ for any $v \in H(\Delta, \Omega)$, we conclude as before that $z \in H(\Delta, \Omega)$. Then,

$$
0=\left\langle\operatorname{tr}^{\Delta}(v), z\right\rangle_{\mathcal{S}}=(v, \Delta z)-(\Delta v, z)=-\left\langle\operatorname{tr}_{\Omega}^{\Delta}(z), v\right\rangle_{\Gamma} \quad \forall v \in H(\Delta, \Omega)
$$

implies that $\operatorname{tr}_{\Omega}^{\Delta}(z)=0$, cf. (6). That is, $z \in H_{0}(\Delta, \Omega)$.

It remains to prove (ii). Here we follow [13, Proof of Proposition 3.8(ii)]. By definition of the norms it holds $\sum_{T \in \mathcal{T}}\left\|\widehat{\boldsymbol{z}}_{T}\right\|_{\Delta, \partial T}^{2} \leq\|\widehat{\boldsymbol{z}}\|_{\Delta, \mathcal{S}}^{2}$ for any $\widehat{\boldsymbol{z}}=\left(\widehat{\boldsymbol{z}}_{T}\right)_{T} \in \mathbf{H}^{\Delta}(\mathcal{S}) \cup \mathbf{H}_{00}^{\Delta}(\mathcal{S})$. To show the other bound let $\widehat{\boldsymbol{z}}=\left(\widehat{\boldsymbol{z}}_{T}\right)_{T} \in \mathbf{H}^{\Delta}(\mathcal{S}) \cup \mathbf{H}_{00}^{\Delta}(\mathcal{S})$ be given with $z \in H(\Delta, \Omega)$ such that $\operatorname{tr}^{\Delta}(z)=\widehat{\boldsymbol{z}}$. Furthermore, for any $T \in \mathcal{T}$, there exists $\tilde{z}_{T} \in H(\Delta, T)$ such that $\operatorname{tr}_{T}^{\Delta}\left(\tilde{z}_{T}\right)=\widehat{\boldsymbol{z}}_{T}$ and $\left\|\tilde{z}_{T}\right\|_{\Delta, T}=\left\|\widehat{\boldsymbol{z}}_{T}\right\|_{\Delta, \partial T}$. Defining $\tilde{z} \in H(\Delta, \mathcal{T})$ by $\left.\tilde{z}\right|_{T}:=\tilde{z}_{T}(T \in \mathcal{T})$ we find with part (i) that $\tilde{z} \in H(\Delta, \Omega)$ with $\operatorname{tr}^{\Delta}(\tilde{z})=\widehat{z}$. Therefore,

$$
\sum_{T \in \mathcal{T}}\left\|\widehat{\boldsymbol{z}}_{T}\right\|_{\Delta, \partial T}^{2}=\sum_{T \in \mathcal{T}}\left\|\tilde{z}_{T}\right\|_{\Delta, T}^{2}=\|\tilde{z}\|_{\Delta}^{2} \geq\|\widehat{\boldsymbol{z}}\|_{\Delta, \mathcal{S}}^{2},
$$

which was left to prove.

Proposition 3. It holds the identity

$$
\|\widehat{\boldsymbol{z}}\|_{\Delta^{\prime}, \mathcal{S}}=\|\widehat{\boldsymbol{z}}\|_{\Delta, \mathcal{S}} \quad \forall \widehat{\boldsymbol{z}} \in \mathbf{H}^{\Delta}(\mathcal{S}) .
$$

In particular,

$$
\operatorname{tr}^{\Delta}: H(\Delta, \Omega) \rightarrow \mathbf{H}^{\Delta}(\mathcal{S}), \quad \operatorname{tr}^{\Delta}: H_{0}(\Delta, \Omega) \rightarrow \mathbf{H}_{00}^{\Delta}(\mathcal{S})
$$

have unit norm and $\mathbf{H}^{\Delta}(\mathcal{S}), \mathbf{H}_{00}^{\Delta}(\mathcal{S})$ are closed.

Proof. Having the tools at hand, the proof is standard (cf., e.g., [1, Theorem 2.3] and [13, Proposition 3.5]). By definition of the involved norms, a duality argument in product spaces, Lemma 1 and Proposition (2) (ii) one finds that

$$
\begin{aligned}
\|\widehat{\boldsymbol{z}}\|_{\Delta^{\prime}, \mathcal{S}}^{2} & =\left(\sup _{0 \neq v \in H(\Delta, \mathcal{T})} \frac{\sum_{T \in \mathcal{T}}\left\langle\widehat{\boldsymbol{z}}_{T}, v\right\rangle_{\partial T}}{\|v\|_{\Delta, \mathcal{T}}}\right)^{2}=\sum_{T \in \mathcal{T}} \sup _{0 \neq v \in H(\Delta, T)} \frac{\left\langle\widehat{\boldsymbol{z}}_{T}, v\right\rangle_{\partial T}^{2}}{\|v\|_{\Delta, T}^{2}} \\
& =\sum_{T \in \mathcal{T}}\left\|\widehat{\boldsymbol{z}}_{T}\right\|_{\Delta^{\prime}, \partial T}^{2}=\sum_{T \in \mathcal{T}}\left\|\widehat{\boldsymbol{z}}_{T}\right\|_{\Delta, \partial T}^{2}=\|\widehat{\boldsymbol{z}}\|_{\Delta, \mathcal{S}}^{2} \quad \forall \widehat{\boldsymbol{z}} \in \mathbf{H}^{\Delta}(\mathcal{S}) .
\end{aligned}
$$

The spaces $\mathbf{H}^{\Delta}(\mathcal{S})$ and $\mathbf{H}_{00}^{\Delta}(\mathcal{S})$ are closed as the images of bounded below operators.

\subsection{Traces and jumps, part two}

As it is not straightforward to discretize the range of $\operatorname{tr}_{T}^{\Delta}$ (where the trace components are coupled), we proceed to introduce different trace operators and spaces. According to the regularity of $u$ (the solution of (1D) and $\Delta u$ (which will be represented by an independent variable) we consider two different cases. 


\subsubsection{Trace of $u$.}

Let us start by defining a trace operator that takes $H^{2}(T)$ instead of $H(\Delta, T)$ as domain $(T \in \mathcal{T})$. It is the restriction of $\operatorname{tr}_{T}^{\Delta}$, cf. (3),

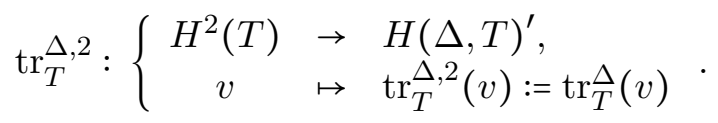

Similarly as before, we have the duality relation

$$
\left\langle\operatorname{tr}_{T}^{\Delta, 2}(v), z\right\rangle_{\partial T}=-\left\langle\operatorname{tr}_{T}^{\Delta}(z), v\right\rangle_{\partial T} \quad \forall v \in H^{2}(T), z \in H(\Delta, T) .
$$

The corresponding collective trace operator (including boundary conditions) is

$$
\operatorname{tr}^{\Delta, 2}:\left\{\begin{array}{ccc}
H_{0}^{2}(\Omega) & \rightarrow H(\Delta, \mathcal{T})^{\prime} \\
v & \mapsto & \operatorname{tr}^{\Delta, 2}(v):=\left(\operatorname{tr}_{T}^{\Delta, 2}(v)\right)_{T}
\end{array}\right.
$$

with duality

$$
\left\langle\operatorname{tr}^{\Delta, 2}(v), z\right\rangle_{\mathcal{S}}:=\sum_{T \in \mathcal{T}}\left\langle\operatorname{tr}_{T}^{\Delta, 2}(v), z\right\rangle_{\partial T} \quad\left(v \in H_{0}^{2}(\Omega), z \in H(\Delta, \mathcal{T})\right)
$$

The ranges of these operators are denoted by

$$
\mathbf{H}^{\Delta, 2}(\partial T):=\operatorname{tr}_{T}^{\Delta, 2}\left(H^{2}(T)\right) \quad(T \in \mathcal{T}) \quad \text { and } \quad \mathbf{H}_{00}^{\Delta, 2}(\mathcal{S}):=\operatorname{tr}^{\Delta, 2}\left(H_{0}^{2}(\Omega)\right) .
$$

As before, the local and global trace spaces are equipped with canonical trace norms,

$$
\begin{array}{rlrl}
\|\widehat{\boldsymbol{v}}\|_{2, \partial T} & :=\inf \left\{\|v\|_{2, T} ; v \in H^{2}(T), \operatorname{tr}_{T}^{\Delta}(v)=\widehat{\boldsymbol{v}}\right\} & & \left(\widehat{\boldsymbol{v}} \in \mathbf{H}^{\Delta, 2}(\partial T), T \in \mathcal{T}\right), \\
\|\widehat{\boldsymbol{v}}\|_{2, \mathcal{S}}:=\inf \left\{\|v\|_{2} ; v \in H_{0}^{2}(\Omega), \operatorname{tr}^{\Delta}(v)=\widehat{\boldsymbol{v}}\right\} & & \left(\widehat{\boldsymbol{v}} \in \mathbf{H}_{00}^{\Delta, 2}(\mathcal{S})\right),
\end{array}
$$

and alternative norms are induced by the respective duality,

$$
\begin{aligned}
\|\widehat{\boldsymbol{v}}\|_{\Delta^{\prime}, \partial T}:=\sup _{0 \neq z \in H(\Delta, T)} \frac{\langle\widehat{\boldsymbol{v}}, z\rangle_{\partial T}}{\|z\|_{\Delta, T}} & \left(\widehat{\boldsymbol{v}} \in \mathbf{H}^{\Delta, 2}(\partial T), T \in \mathcal{T}\right), \\
\|\widehat{\boldsymbol{v}}\|_{\Delta^{\prime}, \mathcal{S}}:=\sup _{0 \neq z \in H(\Delta, \mathcal{T})} \frac{\langle\widehat{\boldsymbol{v}}, z\rangle_{\mathcal{S}}}{\|z\|_{\Delta, \mathcal{T}}} & \left(\widehat{\boldsymbol{v}} \in \mathbf{H}_{00}^{\Delta, 2}(\mathcal{S})\right) .
\end{aligned}
$$

It goes without saying that the dualities on $\partial T$ and $\mathcal{S}$ are defined by the corresponding trace operations (3) (generically for any local space), and (11) on $\mathcal{S}$. For instance, the duality $\langle\widehat{\boldsymbol{v}}, z\rangle_{\partial T}$ between $\widehat{\boldsymbol{v}} \in \mathbf{H}^{\Delta, 2}(\partial T)$ and $z \in H(\Delta, T)$ is $(\Delta z, v)_{T}-(z, \Delta v)_{T}$ with arbitrary $v \in H^{2}(T)$ such that $\operatorname{tr}_{T}^{\Delta, 2}(v)=\widehat{\boldsymbol{v}}$.

It is immediate that all the trace operators are bounded both with respect to the respective canonical trace norm and the respective duality norm. 
Remark 4. The trace operator $\operatorname{tr}_{T}^{\Delta, 2}$ gives rise to two components, $\operatorname{tr}_{T}^{\Delta, 2}(v)=\left(\left.v\right|_{\partial T},\left.\partial_{\mathbf{n}} v\right|_{\partial T}\right)$ for $v \in H^{2}(T)$. On a non-smooth boundary $\partial T$, they are generally not independent. That is, this trace operator does not map surjectively onto the product space of separate traces, $\left.v\right|_{\partial T}$ and $\left.\partial_{\mathbf{n}} v\right|_{\partial T}, c f$. Grisvard [17]. In [6], Costabel and Dauge discuss this subject including dual spaces.

Proposition 5. It holds the identity

$$
\|\widehat{\boldsymbol{v}}\|_{\Delta^{\prime}, \mathcal{S}}=\|\widehat{\boldsymbol{v}}\|_{2, \mathcal{S}} \quad \forall \widehat{\boldsymbol{v}} \in \mathbf{H}_{00}^{\Delta, 2}(\mathcal{S}) .
$$

In particular,

$$
\operatorname{tr}^{\Delta, 2}: H_{0}^{2}(\Omega) \rightarrow \mathbf{H}_{00}^{\Delta, 2}(\mathcal{S})
$$

has unit norm and $\mathbf{H}_{00}^{\Delta, 2}(\mathcal{S})$ is closed.

Proof. Let $\widehat{\boldsymbol{v}}=\left(\widehat{\boldsymbol{v}}_{T}\right)_{T} \in \mathbf{H}_{00}^{\Delta, 2}(\mathcal{S})$ be given. By definition of the norms, one sees that $\|\widehat{\boldsymbol{v}}\|_{\Delta^{\prime}, \mathcal{S}} \leq$ $\|v\|_{\Delta}$ for any $v \in H_{0}^{2}(\Omega)$ with $\operatorname{tr}^{\Delta, 2}(v)=\widehat{\boldsymbol{v}}$. Since

$$
\|\Delta v\|=\|\varepsilon \nabla v\| \quad \forall v \in H_{0}^{2}(\Omega)
$$

(cf. [2, (1.2.8)]) we conclude that $\|\widehat{\boldsymbol{v}}\|_{\Delta^{\prime}, \mathcal{S}} \leq\|\widehat{\boldsymbol{v}}\|_{2, \mathcal{S}}$.

To show the other inequality, we define $v_{T} \in H^{2}(T)(T \in \mathcal{T})$ as the solution to

$$
\left(\operatorname{div} \operatorname{div} \varepsilon \nabla v_{T}+v_{T}=\right) \Delta^{2} v_{T}+v_{T}=0 \quad \text { in } \quad T, \quad \operatorname{tr}_{T}^{\Delta, 2}\left(v_{T}\right)=\widehat{\boldsymbol{v}}_{T},
$$

and introduce functions $v, z$ with $\left.v\right|_{T}=v_{T}$ and $\left.z\right|_{T}=\Delta v_{T}(T \in \mathcal{T})$. We conclude that $v \in H_{0}^{2}(\Omega)$ and $\|v\|_{2}=\|\widehat{\boldsymbol{v}}\|_{2, \mathcal{S}}$. Furthermore, since $\Delta z_{T}=-v_{T}, z \in H(\Delta, \mathcal{T})$, and also using relation (12) we find that

$$
\|z\|_{\Delta, \mathcal{T}}^{2}=\sum_{T \in \mathcal{T}}\left\|\Delta v_{T}\right\|_{T}^{2}+\left\|v_{T}\right\|_{T}^{2}=\|v\|_{\Delta}^{2}=\|v\|_{2}^{2}
$$

Finally, we observe that

$$
\|v\|_{2}^{2}=\|v\|_{\Delta}^{2}=\sum_{T \in \mathcal{T}}\left(\Delta v_{T}, \Delta v_{T}\right)_{T}+\left(v_{T}, v_{T}\right)_{T}=\sum_{T \in \mathcal{T}}-\left\langle\operatorname{tr}_{T}^{\Delta, 2}\left(v_{T}\right), \Delta v_{T}\right\rangle_{\partial T}=-\langle\widehat{\boldsymbol{v}}, z\rangle_{\mathcal{S}}
$$

Here, we made use of the relation $\Delta^{2} v_{T}+v_{T}=0$. Collecting the findings we conclude that

$$
\|\widehat{\boldsymbol{v}}\|_{2, \mathcal{S}}^{2}=\|v\|_{2}^{2}=\|z\|_{\Delta, \mathcal{T}}^{2}=-\langle\widehat{\boldsymbol{v}}, z\rangle_{\mathcal{S}} .
$$

This yields

$$
\|\widehat{\boldsymbol{v}}\|_{2, \mathcal{S}} \leq\|\widehat{\boldsymbol{v}}\|_{\Delta^{\prime}, \mathcal{S}}
$$

and finishes the proof.

Remark 6. Comparing the results for our trace operators $\operatorname{tr}^{\Delta}$ (Proposition 3) and $\operatorname{tr}^{\Delta, 2}$ (Proposition 5) one notices that there is no result for the local operator $\operatorname{tr}_{T}^{\Delta, 2}$ that corresponds to Lemma 1. The reason for the lack of such a local property is that relation 12 requires homogeneous boundary conditions. 
Proposition 7. For $z \in H(\Delta, \mathcal{T})$ it holds

$$
z \in H(\Delta, \Omega) \quad \Leftrightarrow \quad\left\langle\operatorname{tr}^{\Delta, 2}(v), z\right\rangle_{\mathcal{S}}=0 \quad \forall v \in H_{0}^{2}(\Omega)
$$

Proof. The proof is analogous to that of Proposition 2(i). The direction " $\Rightarrow$ " follows by integration by parts and density arguments. The other direction is proved by taking $z \in H(\Delta, \mathcal{T})$ with $\left\langle\operatorname{tr}^{\Delta, 2}(v), z\right\rangle_{\mathcal{S}}=0$ for any $v \in H_{0}^{2}(\Omega)$, and concluding that $\Delta z \in L_{2}(\Omega)$ so that $z \in H(\Delta, \Omega)$.

\subsubsection{Trace of $\Delta u$.}

Now let us turn to possible trace operations for $\Delta u$ ( $u$ representing a function with a regularity according to the solution of (1)). Obviously, since $f \in L_{2}(\Omega)$ by assumption, $\Delta u \in H(\Delta, \Omega)$ by (1a). That is why we have considered the trace operator $\operatorname{tr}^{\Delta}$ in $\$ 3.2$. Since we have restricted the domain for the definition of $\operatorname{tr}^{\Delta, 2}$, duality considerations reveal that we now have to consider extended traces by testing with $H^{2}$-functions. This seems to force to define an operator

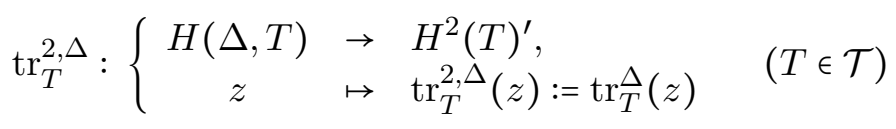

with corresponding collective trace operator $\operatorname{tr}^{2, \Delta}$, and trace norms and norms defined by duality with $H^{2}$. Again, this operator gives rise to two components,

$$
\left.(\cdot)\right|_{\partial T}:\left\{\begin{array}{cccc}
H(\Delta, T) & \rightarrow & \left\{z \in H^{2}(T) ;\left.\partial_{\mathbf{n}} z\right|_{\partial T}=0\right\}^{\prime} \\
v & \mapsto & z \mapsto\left\langle\operatorname{tr}_{T}^{2, \Delta}(v), z\right\rangle_{\partial T}
\end{array} \quad(T \in \mathcal{T})\right.
$$

and

$$
\left.\left(\partial_{\mathbf{n}} \cdot\right)\right|_{\partial T}:\left\{\begin{array}{ccc}
H(\Delta, T) & \rightarrow & \left\{z \in H^{2}(T) ;\left.z\right|_{\partial T}=0\right\}^{\prime} \\
v & \mapsto & z \mapsto-\left\langle\operatorname{tr}_{T}^{2, \Delta}(v), z\right\rangle_{\partial T}
\end{array} \quad(T \in \mathcal{T}),\right.
$$

cf. (4). For a smooth boundary $\partial T$, the two components are independent as in that case the operator $\operatorname{tr}_{T}^{2, \Delta}$ maps $H(\Delta, T)$ onto $H^{-3 / 2}(\partial T) \times H^{-1 / 2}(\partial T):=H^{3 / 2}(\partial T)^{\prime} \times H^{1 / 2}(\partial T)^{\prime}$. Here, $H^{3 / 2}(\partial T)$ denotes the space of traces onto $\partial T$ of $H^{2}(T)$-functions, and $H^{1 / 2}(\partial T)$ is that of the normal derivatives. Glowinski and Pironneau give details in [16, Props 2.3, 2.4] and refer to Lions and Magenes for a proof, see [19, Chapter 2: Theorem 6.5, Section 9.8 (p. 213)]. However, on a polygonal element $T$, the trace operator is not surjective onto $H^{-3 / 2}(\partial T) \times H^{-1 / 2}(\partial T)$. This has been indicated by Costabel and Dauge in [6]. Furthermore, it turns out that in general the operator $\operatorname{tr}_{T}^{2, \Delta}$ is not bounded below. We give a counterexample in the appendix.

For these reasons we avoid to employ the seemingly obvious choice (13). Instead, we take a trace operator defined in [13]. It can be interpreted as an extension of $\operatorname{tr}_{T}^{2, \Delta}$ to a larger domain, see Lemma 10 below. Let us repeat some definitions and needed properties from [13].

We introduce trace operators $\operatorname{tr}_{T}^{\mathrm{dDiv}}: H(\operatorname{div} \operatorname{div}, T) \rightarrow H^{2}(T)^{\prime}$ for $T \in \mathcal{T}$ by

$$
\left\langle\operatorname{tr}_{T}^{\mathrm{dDiv}}(\boldsymbol{\Theta}), z\right\rangle_{\partial T}:=(\operatorname{div} \operatorname{div} \boldsymbol{\Theta}, z)_{T}-(\boldsymbol{\Theta}, \varepsilon \nabla z)_{T}
$$


with the collective variant defined as

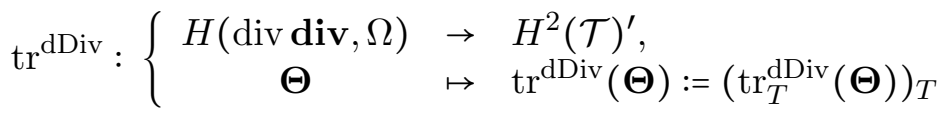

with duality

$$
\left\langle\operatorname{tr}^{\mathrm{dDiv}}(\boldsymbol{\Theta}), z\right\rangle_{\mathcal{S}}:=\sum_{T \in \mathcal{T}}\left\langle\operatorname{tr}_{T}^{\mathrm{dDiv}}(\boldsymbol{\Theta}), z\right\rangle_{\partial T}
$$

The range of $\operatorname{tr}^{\mathrm{dDiv}}$ is denoted by

$$
\mathbf{H}^{-3 / 2,-1 / 2}(\mathcal{S}):=\operatorname{tr}^{\mathrm{d} \operatorname{Div}}(H(\operatorname{div} \operatorname{div}, \Omega))
$$

and provided with the trace norm

$$
\|\widehat{\boldsymbol{q}}\|_{\mathrm{dDiv}, \mathcal{S}}:=\inf \left\{\|\boldsymbol{\Theta}\|_{\operatorname{div} \operatorname{div}} ; \boldsymbol{\Theta} \in H(\operatorname{div} \operatorname{div}, \Omega), \operatorname{tr}^{\mathrm{dDiv}}(\boldsymbol{\Theta})=\widehat{\boldsymbol{q}}\right\}
$$

or the duality norm

$$
\|\widehat{\boldsymbol{q}}\|_{-3 / 2,-1 / 2, \mathcal{S}}:=\sup _{0 \neq z \in H^{2}(\mathcal{T})} \frac{\langle\widehat{\boldsymbol{q}}, z\rangle_{\mathcal{S}}}{\|z\|_{2, \mathcal{T}}}, \quad \widehat{\boldsymbol{q}} \in \mathbf{H}^{-3 / 2,-1 / 2}(\mathcal{S}) .
$$

Here, the duality is defined as

$$
\langle\widehat{\boldsymbol{q}}, z\rangle_{\mathcal{S}}:=\sum_{T \in \mathcal{T}}\left\langle\widehat{\boldsymbol{q}}_{T}, z\right\rangle_{\partial T}
$$

with

$$
\langle\widehat{\boldsymbol{q}}, z\rangle_{\partial T}:=\left\langle\operatorname{tr}_{T}^{\mathrm{dDiv}}(\boldsymbol{\Theta}), z\right\rangle_{\partial T} \quad \text { for } \boldsymbol{\Theta} \in H(\operatorname{div} \operatorname{div}, T) \text { with } \operatorname{tr}_{T}^{\mathrm{dDiv}}(\boldsymbol{\Theta})=\widehat{\boldsymbol{q}}=\left(\widehat{\boldsymbol{q}}_{T}\right)_{T},
$$

as in (14) and (15).

Proposition 8 ([13, Proposition 5]). It holds the identity

$$
\|\widehat{\boldsymbol{q}}\|_{-3 / 2,-1 / 2, \mathcal{S}}=\|\widehat{\boldsymbol{q}}\|_{\mathrm{dDiv}, \mathcal{S}} \quad \forall \widehat{\boldsymbol{q}} \in \mathbf{H}^{-3 / 2,-1 / 2}(\mathcal{S}) .
$$

In particular,

$$
\operatorname{tr}^{\mathrm{dDiv}}: H(\operatorname{div} \operatorname{div}, \Omega) \rightarrow \mathbf{H}^{-3 / 2,-1 / 2}(\mathcal{S})
$$

has unit norm and $\mathbf{H}^{-3 / 2,-1 / 2}(\mathcal{S})$ is closed.

Proposition 9 ([13, Proposition 8]). For $z \in H^{2}(\mathcal{T})$ the following equivalence holds,

$$
z \in H_{0}^{2}(\Omega) \quad \Leftrightarrow \quad\langle\widehat{\boldsymbol{q}}, z\rangle_{\mathcal{S}}=0 \quad \forall \widehat{\boldsymbol{q}} \in \mathbf{H}^{-3 / 2,-1 / 2}(\mathcal{S}) .
$$


Now, the connection between $\operatorname{tr}^{2, \Delta}$ and $\operatorname{tr}^{\mathrm{dDiv}}$ is as follows. For given $\sigma \in H(\Delta, \Omega)$, it holds $\mathbb{D}(\sigma):=\left(\begin{array}{ll}\sigma & 0 \\ 0 & \sigma\end{array}\right) \in H(\operatorname{div} \operatorname{div}, \Omega)$ since $\operatorname{div} \operatorname{div} \mathbb{D}(\sigma)=\Delta \sigma$, and one concludes that

$$
\begin{aligned}
\left\langle\operatorname{tr}^{2, \Delta}(\sigma), v\right\rangle_{\mathcal{S}} & =(\sigma, \Delta v)_{\mathcal{T}}-(\Delta \sigma, v) \\
& =(\mathbb{D}(\sigma), \varepsilon \nabla v)_{\mathcal{T}}-(\operatorname{div} \operatorname{div} \mathbb{D}(\sigma), v)=-\left\langle\operatorname{tr}^{\mathrm{dDiv}}(\mathbb{D}(\sigma)), v\right\rangle_{\mathcal{S}} \quad \forall v \in H^{2}(\mathcal{T}) .
\end{aligned}
$$

It is clear that $\mathbb{D}: H(\Delta, \Omega) \rightarrow H(\operatorname{div} \operatorname{div}, \Omega)$ is not surjective. Furthermore, since traces of images of $\mathbb{D}$ do not have jump terms at vertices of $\mathcal{T}$ which are present in the case of traces of $H(\operatorname{div} \operatorname{div}, \Omega)$, see [13], it is clear that $\operatorname{tr}^{2, \Delta}$ does not map surjectively onto $\mathbf{H}^{-3 / 2,-1 / 2}(\mathcal{S})$.

Let us note this result.

\section{Lemma 10.}

$$
\operatorname{tr}^{2, \Delta}=-\operatorname{tr}^{\mathrm{dDiv}} \circ \mathbb{D}: H(\Delta, \Omega) \rightarrow \mathbf{H}^{-3 / 2,-1 / 2}(\mathcal{S})
$$

is bounded but not surjective. In particular, Dirac distributions at boundary points, $\delta_{e}: z \mapsto$ $\left.z\right|_{T}(e)\left(e \in \Gamma \cap \bar{T}, T \in \mathcal{T}, z \in H^{2}(\mathcal{T})\right.$ with $\left.\operatorname{supp}(z)=\bar{T}\right)$ are elements of $\mathbf{H}^{-3 / 2,-1 / 2}(\mathcal{S})$ but not of $\operatorname{tr}^{2, \Delta}(H(\Delta, \Omega))$.

The fact that $\delta_{e} \notin \operatorname{tr}^{2, \Delta}(H(\Delta, \Omega))$ is illustrated in Appendix $\mathrm{B}$.

\section{First variational formulation and DPG approximation}

Let us continue to develop a variational formulation of (1). Considering the trace operator $\operatorname{tr}^{\Delta}$ from $\$ 3.2$, our preliminary formulation (2) now reads

$$
(u, \Delta \tau)_{\mathcal{T}}+(\sigma, \Delta v-\tau)_{\mathcal{T}}-\left\langle\operatorname{tr}^{\Delta}(\sigma), v\right\rangle_{\mathcal{S}}-\left\langle\operatorname{tr}^{\Delta}(u), \tau\right\rangle_{\mathcal{S}}=(f, v) .
$$

In this case, test functions $v$ and $\tau$ come from $H(\Delta, \mathcal{T})$. Therefore, introducing independent trace variables $\widehat{\boldsymbol{\sigma}}:=\operatorname{tr}^{\Delta}(\sigma), \widehat{\boldsymbol{u}}:=\operatorname{tr}^{\Delta}(u)$, and spaces

$$
\mathcal{U}_{1}:=L_{2}(\Omega) \times L_{2}(\Omega) \times \mathbf{H}_{00}^{\Delta}(\mathcal{S}) \times \mathbf{H}^{\Delta}(\mathcal{S}), \quad \mathcal{V}_{1}:=H(\Delta, \mathcal{T}) \times H(\Delta, \mathcal{T})
$$

with respective norms

$$
\|(u, \sigma, \widehat{\boldsymbol{u}}, \widehat{\boldsymbol{\sigma}})\|_{\mathcal{U}_{1}}^{2}:=\|u\|^{2}+\|\sigma\|^{2}+\|\widehat{\boldsymbol{u}}\|_{\Delta, \mathcal{S}}^{2}+\|\widehat{\boldsymbol{\sigma}}\|_{\Delta, \mathcal{S}}^{2}, \quad\|(v, \tau)\|_{\mathcal{V}_{1}}^{2}:=\|v\|_{\Delta, \mathcal{T}}^{2}+\|\tau\|_{\Delta, \mathcal{T}}^{2},
$$

our first ultraweak variational formulation of (1) is

$$
(u, \sigma, \widehat{\boldsymbol{u}}, \widehat{\boldsymbol{\sigma}}) \in \mathcal{U}_{1}: \quad b_{1}(u, \sigma, \widehat{\boldsymbol{u}}, \widehat{\boldsymbol{\sigma}} ; v, \tau)=L(v, \tau) \quad \forall(v, \tau) \in \mathcal{V}_{1},
$$

in strong form written as $B_{1}(u, \sigma, \widehat{\boldsymbol{u}}, \widehat{\boldsymbol{\sigma}})=L \in \mathcal{V}_{1}^{\prime}$. Here,

$$
b_{1}(u, \sigma, \widehat{\boldsymbol{u}}, \widehat{\boldsymbol{\sigma}} ; v, \tau):=(u, \Delta \tau)_{\mathcal{T}}+(\sigma, \Delta v-\tau)_{\mathcal{T}}-\langle\widehat{\boldsymbol{u}}, \tau\rangle_{\mathcal{S}}-\langle\widehat{\boldsymbol{\sigma}}, v\rangle_{\mathcal{S}}
$$

$L(v, \tau):=(f, v)$, and $\langle\cdot, \cdot\rangle_{\mathcal{S}}$ refers to the duality between $\mathbf{H}^{\Delta}(\mathcal{S})$ (including $\left.\mathbf{H}_{00}^{\Delta}(\mathcal{S})\right)$ and $H(\Delta, \mathcal{T})$ implied by $(5)$. 
Theorem 11. The operator $B_{1}: \mathcal{U}_{1} \rightarrow \mathcal{V}_{1}^{\prime}$ is continuous and bounded below. In particular, for any function $f \in L_{2}(\Omega)$, there exists a unique and stable solution $(u, \sigma, \widehat{\boldsymbol{u}}, \widehat{\boldsymbol{\sigma}}) \in \mathcal{U}_{1}$ to (17),

$$
\|u\|+\|\sigma\|+\|\widehat{\boldsymbol{u}}\|_{\Delta, \mathcal{S}}+\|\widehat{\boldsymbol{\sigma}}\|_{\Delta, \mathcal{S}} \lesssim\|f\|
$$

with a hidden constant that is independent of $f$ and $\mathcal{T}$. Furthermore, (1) and (17) are equivalent: If $u \in H_{0}^{2}(\Omega)$ solves (1) then $\mathbf{u}:=\left(u, \Delta u, \operatorname{tr}^{\Delta}(u), \operatorname{tr}^{\Delta}(\Delta u)\right)$ solves 17); and if $\mathbf{u}=(u, \sigma, \widehat{\boldsymbol{u}}, \widehat{\boldsymbol{\sigma}})$ solves (17) then $u$ is element of $H_{0}^{2}(\Omega)$ and solves (1).

For a proof of this theorem we refer to Section 6 .

A DPG approximation with optimal test functions based on formulation (17) is as follows. We select discrete spaces $\mathcal{U}_{1, h} \subset \mathcal{U}$ and test spaces $\mathcal{V}_{1, h}:=\mathrm{T}_{1}\left(\mathcal{U}_{1, h}\right) \subset \mathcal{V}_{1}$ where $\mathrm{T}_{1}: \mathcal{U}_{1} \rightarrow \mathcal{V}_{1}$ is the trial-to-test operator defined by

$$
\left\langle\left\langle\mathrm{T}_{1}(\mathbf{u}), \mathbf{v}\right\rangle\right\rangle_{\mathcal{V}_{1}}=b_{1}(\mathbf{u}, \mathbf{v}) \quad \forall \mathbf{v} \in \mathcal{V}_{1}
$$

Here, $\left\langle\langle\cdot, \cdot\rangle_{\mathcal{V}_{1}}\right.$ is the inner product in $\mathcal{V}_{1}$ that generates the selected norm $\left(\|\cdot\|_{\Delta, \mathcal{T}}^{2}+\|\cdot\|_{\Delta, \mathcal{T}}^{2}\right)^{1 / 2}$.

Then, an approximation $\mathbf{u}_{h}=\left(u_{h}, \sigma_{h}, \widehat{\boldsymbol{u}}_{h}, \widehat{\boldsymbol{\sigma}}_{h}\right) \in \mathcal{U}_{1, h}$ is defined as the solution to

$$
b_{1}\left(\mathbf{u}_{h}, \mathbf{v}\right)=L(\mathbf{v}) \quad \forall \mathbf{v} \in \mathcal{V}_{1, h} .
$$

Being a minimum residual method it delivers the best approximation of the exact solution in the residual norm $\left\|B_{1}(\cdot)\right\|_{\mathcal{V}_{1}^{\prime}}$, cf., e.g., [8]. Then, using the equivalence of the norms $\left\|B_{1}(\cdot)\right\|_{\mathcal{V}_{1}^{\prime}}$ and $\|\cdot\|_{\mathcal{U}_{1}}$ stated by Theorem 11, we obtain its quasi-optimal convergence in the latter norm.

Theorem 12. Let $f \in L_{2}(\Omega)$ be given and let $\mathbf{u}$ be the solution of (17). For any finitedimensional subspace $\mathcal{U}_{1, h} \subset \mathcal{U}_{1}$ there exists a unique solution $\mathbf{u}_{h} \in \mathcal{U}_{1, h}$ to (19). It satisfies the quasi-optimal error estimate

$$
\left\|\mathbf{u}-\mathbf{u}_{h}\right\|_{\mathcal{U}_{1}} \lesssim\|\mathbf{u}-\mathbf{w}\|_{\mathcal{U}_{1}} \quad \forall \mathbf{w} \in \mathcal{U}_{1, h}
$$

with a hidden constant that is independent of $f, \mathcal{T}$ and $\mathcal{U}_{1, h}$.

\section{Second variational formulation and DPG approximation}

Let us reconsider the preliminary formulation (2). We make use of the regularity $u \in H_{0}^{2}(\Omega)$. Then, the variable $\widehat{\boldsymbol{u}}$ replaces $\operatorname{tr}^{\Delta, 2}(u) \in \mathbf{H}_{00}^{\Delta, 2}(\mathcal{S})$ instead of $\operatorname{tr}^{\Delta}(u) \in \mathbf{H}_{00}^{\Delta}(\mathcal{S})$. We then use test functions $v \in H^{2}(\mathcal{T})$ instead of $v \in H(\Delta, \mathcal{T})$. This means that we have a trace $\operatorname{tr}^{\mathrm{dDiv}} \circ \mathbb{D}(\Delta u) \in$ $\mathbf{H}^{-3 / 2,-1 / 2}(\mathcal{S})$ (cf. Lemma 10 rather than $\operatorname{tr}^{\Delta}(\Delta u) \in \mathbf{H}^{\Delta}(\mathcal{S})$. This corresponds to using the spaces

$$
\mathcal{U}_{2}:=L_{2}(\Omega) \times L_{2}(\Omega) \times \mathbf{H}_{00}^{\Delta, 2}(\mathcal{S}) \times \mathbf{H}^{-3 / 2,-1 / 2}(\mathcal{S}), \quad \mathcal{V}_{2}:=H^{2}(\mathcal{T}) \times H(\Delta, \mathcal{T})
$$

with respective norms

$$
\|(u, \sigma, \widehat{\boldsymbol{u}}, \widehat{\boldsymbol{\sigma}})\|_{\mathcal{U}_{2}}^{2}:=\|u\|^{2}+\|\sigma\|^{2}+\|\widehat{\boldsymbol{u}}\|_{2, \mathcal{S}}^{2}+\|\widehat{\boldsymbol{\sigma}}\|_{\mathrm{dDiv}, \mathcal{S}}^{2}, \quad\|(v, \tau)\|_{\mathcal{V}_{2}}^{2}:=\|v\|_{2, \mathcal{T}}^{2}+\|\tau\|_{\Delta, \mathcal{T}}^{2} .
$$


The corresponding ultraweak variational formulation is:

$$
(u, \sigma, \widehat{\boldsymbol{u}}, \widehat{\boldsymbol{\sigma}}) \in \mathcal{U}_{2}: \quad b_{2}(u, \sigma, \widehat{\boldsymbol{u}}, \widehat{\boldsymbol{\sigma}} ; v, \tau)=L(v, \tau) \quad \forall(v, \tau) \in \mathcal{V}_{2} .
$$

Here, the bilinear form $b_{2}$ is defined similarly as $b_{1}$ in (18), namely

$$
b_{2}(u, \sigma, \widehat{\boldsymbol{u}}, \widehat{\boldsymbol{\sigma}} ; v, \tau):=(u, \Delta \tau)_{\mathcal{T}}+(\sigma, \Delta v-\tau)_{\mathcal{T}}-\langle\widehat{\boldsymbol{u}}, \tau\rangle_{\mathcal{S}}+\langle\widehat{\boldsymbol{\sigma}}, v\rangle_{\mathcal{S}}
$$

Specifically, the duality $\langle\widehat{\boldsymbol{u}}, \tau\rangle_{\mathcal{S}}$ is the one induced by $(11)$ analogously as before, and $\langle\widehat{\boldsymbol{\sigma}}, v\rangle_{\mathcal{S}}$ is defined by (16). For consistency with trace definitions we have changed the sign in front of the latter duality, cf. Lemma 10.

We refer to the strong form of 200 as $B_{2}(u, \sigma, \widehat{\boldsymbol{u}}, \widehat{\boldsymbol{\sigma}})=L \in \mathcal{V}_{2}^{\prime}$.

Theorem 13. The operator $B_{2}: \mathcal{U}_{2} \rightarrow \mathcal{V}_{2}^{\prime}$ is continuous and bounded below. In particular, for any function $f \in L_{2}(\Omega)$, there exists a unique solution $(u, \sigma, \widehat{\boldsymbol{u}}, \widehat{\boldsymbol{\sigma}})$ of $(20)$. It holds the bound

$$
\|u\|+\|\sigma\|+\|\widehat{\boldsymbol{u}}\|_{2, \mathcal{S}}+\|\widehat{\boldsymbol{\sigma}}\|_{\mathrm{dDiv}, \mathcal{S}} \lesssim\|f\|
$$

with a hidden constant that is independent of $f$ and $\mathcal{T}$. Furthermore, (1) and (20) are equivalent: If $u \in H_{0}^{2}(\Omega)$ solves (1) then $\mathbf{u}:=\left(u, \Delta u, \operatorname{tr}^{\Delta, 2}(u), \operatorname{tr}^{\mathrm{dDiv}}(\mathbb{D}(\Delta u))\right)$ solves (20); and if $\mathbf{u}=(u, \sigma, \widehat{\boldsymbol{u}}, \widehat{\boldsymbol{\sigma}})$ solves 20 then $u \in H_{0}^{2}(\Omega)$ solves (1).

A proof of this result is given in Section 6 .

The corresponding DPG approximation uses discrete spaces $\mathcal{U}_{2, h} \subset \mathcal{U}_{2}$ and test spaces $\mathcal{V}_{2, h}:=$ $\mathrm{T}_{2}\left(\mathcal{U}_{2, h}\right) \subset \mathcal{V}_{2}$ where the trial-to-test operator $\mathrm{T}_{2}: \mathcal{U}_{2} \rightarrow \mathcal{V}_{2}$ is defined by

$$
\left\langle\left\langle\mathrm{T}_{2}(\mathbf{u}), \mathbf{v}\right\rangle\right\rangle_{\mathcal{V}_{2}}=b_{2}(\mathbf{u}, \mathbf{v}) \quad \forall \mathbf{v} \in \mathcal{V}_{2}
$$

with inner product $\langle\cdot \cdot \cdot \cdot\rangle_{\mathcal{V}_{2}}$ that induces the norm $\left(\|\cdot\|_{2, \mathcal{T}^{+}}^{2}\|\cdot\|_{\Delta, \mathcal{T}}^{2}\right)^{1 / 2}$ in $\mathcal{V}_{2}$. The approximation $\mathbf{u}_{h}=\left(u_{h}, \sigma_{h}, \widehat{\boldsymbol{u}}_{h}, \widehat{\boldsymbol{\sigma}}_{h}\right) \in \mathcal{U}_{2, h}$ is defined analogously as before,

$$
b_{2}\left(\mathbf{u}_{h}, \mathbf{v}\right)=L(\mathbf{v}) \quad \forall \mathbf{v} \in \mathcal{V}_{2, h}
$$

Again, this scheme converges quasi-optimally, see Theorem 12

Theorem 14. Let $f \in L_{2}(\Omega)$ be given and let $\mathbf{u}$ be the solution of 20 . For any finitedimensional subspace $\mathcal{U}_{2, h} \subset \mathcal{U}_{2}$ there exists a unique DPG approximation $\mathbf{u}_{h}=\left(u_{h}, \sigma_{h}, \widehat{\boldsymbol{u}}_{h}, \widehat{\boldsymbol{\sigma}}_{h}\right) \epsilon$ $\mathcal{U}_{2, h}$ defined by 21]. It satisfies the quasi-optimal error estimate

$$
\left\|\mathbf{u}-\mathbf{u}_{h}\right\|_{\mathcal{U}_{2}} \lesssim\|\mathbf{u}-\mathbf{w}\|_{\mathcal{U}_{2}} \quad \forall \mathbf{w} \in \mathcal{U}_{2, h}
$$

with a hidden constant that is independent of $f, \mathcal{T}$ and $\mathcal{U}_{2, h}$. 


\section{Proofs of Theorems 11 and 13}

We start by showing unique and stable solvability of the (self) adjoint problem to (1), with continuous spaces.

Lemma 15. For given $g_{1}, g_{2} \in L_{2}(\Omega)$, there exists a unique solution $(v, \tau) \in H_{0}^{2}(\Omega) \times H(\Delta, \Omega)$ of

$$
\begin{aligned}
\Delta v-\tau=g_{1} & \text { in } \Omega, \\
\Delta \tau=g_{2} & \text { in } \Omega .
\end{aligned}
$$

It satisfies

$$
\|v\|_{2}+\|\tau\|_{\Delta} \lesssim\left\|g_{1}\right\|+\left\|g_{2}\right\|
$$

with a constant that is independent of $g_{1}, g_{2}$ and $\mathcal{T}$.

Proof. We write a variational formulation for $v$. Applying $\Delta$ to $22 \mathrm{a}$ ) and using (22b), this gives the relation

$$
\Delta\left(\Delta v-g_{1}\right)=g_{2} \quad \text { in } \quad L_{2}(\Omega) .
$$

Testing with $\delta v \in H_{0}^{2}(\Omega)$ and integrating by parts we see that $v \in H_{0}^{2}(\Omega)$ solves

$$
(\Delta v, \Delta \delta v)=\left(g_{1}, \Delta \delta v\right)+\left(g_{2}, \delta v\right) \quad \forall \delta v \in H_{0}^{2}(\Omega) .
$$

By standard arguments, this problem has a unique solution with bound

$$
\|\varepsilon \nabla v\|^{2}=\|\Delta v\|^{2} \leq\left(\left\|g_{1}\right\|^{2}+\left\|g_{2}\right\|^{2}\right)^{1 / 2}\|v\|_{\Delta}
$$

Here, we made use of (12). Using Poincaré's inequality $\|v\| \lesssim\|\varepsilon \nabla v\|$ we conclude that

$$
\|v\|_{2} \lesssim\left\|g_{1}\right\|+\left\|g_{2}\right\|
$$

A unique solution $(v, \tau)$ of $(22)$ is then obtained by setting $\tau:=\Delta v-g_{1}$, with bound

$$
\|\tau\|+\|\Delta \tau\|=\left\|\Delta v-g_{1}\right\|+\left\|g_{2}\right\| \lesssim\left\|g_{1}\right\|+\left\|g_{2}\right\|
$$

This finishes the proof.

\subsection{Proof of Theorem 11 .}

Well-posedness of (17). We check the standard conditions. The boundedness of $b_{1}$ and $L$ holds by definition of the norms in $\mathcal{U}_{1}$ and $\mathcal{V}_{1}$.

The injectivity of the adjoint operator $B_{1}^{*}: \mathcal{V}_{1} \rightarrow \mathcal{U}_{1}^{\prime}$ can be seen as follows. Let $(v, \tau) \in \mathcal{V}_{1}$ be such that $b_{1}(\mathbf{u} ; v, \tau)=0$ for any $\mathbf{u}=(u, \sigma, \widehat{\boldsymbol{u}}, \widehat{\boldsymbol{\sigma}}) \in \mathcal{U}_{1}$. The selection of $\mathbf{u}=(0,0, \widehat{\boldsymbol{u}}, 0)$ for any $\widehat{\boldsymbol{u}} \in \mathbf{H}_{00}^{\Delta}(\mathcal{S})$ reveals that $\tau \in H(\Delta, \Omega)$ by Proposition $2(\mathrm{i})$. Analogously, selecting $\mathbf{u}=$ $(0,0,0, \widehat{\boldsymbol{\sigma}})$ with arbitrary $\widehat{\boldsymbol{\sigma}} \in \mathbf{H}^{\Delta}(\mathcal{S})$, Proposition 2 (i) shows that $v \in H_{0}(\Delta, \Omega)$. We conclude 
that $(v, \tau) \in H_{0}(\Delta, \Omega) \times H(\Delta, \Omega)$ solves $\tau=\Delta v$ and $\Delta \tau=0$. It follows that $\Delta^{2} v=0$. Since $v \in H_{0}(\Delta, \Omega)$, so that $\operatorname{tr}_{\Omega}^{\Delta}(v)=0$, relation (6) shows that $\|\Delta v\|^{2}=\left(\Delta^{2} v, v\right)=0$. In particular, $\tau=\Delta v=0$. Now, defining $z \in H_{0}^{2}(\Omega)$ as the solution to $\Delta^{2} z=v$, and again using (6), we find that $(v, v)=\left(v, \Delta^{2} z\right)=(\Delta v, \Delta z)=0$, that is, $v=0$.

It remains to check the inf-sup condition

$$
\left\|B_{1} \mathbf{u}\right\|_{\mathcal{V}_{1}^{\prime}} \gtrsim\|\mathbf{u}\|_{\mathcal{U}_{1}} \quad \forall \mathbf{u} \in \mathcal{U}_{1}
$$

To this end we employ the technique proposed by Carstensen et al. in [1]. To simplify reading let use relate our notation to the one in [1].

$$
\begin{aligned}
& X=\mathcal{U}_{1}, \quad X_{0}=L_{2}(\Omega) \times L_{2}(\Omega), \quad \hat{X}=\mathbf{H}_{00}^{\Delta}(\mathcal{S}) \times \mathbf{H}^{\Delta}(\mathcal{S}), \\
& Y=\mathcal{V}_{1}, \quad Y_{0}=H_{0}(\Delta, \Omega) \times H(\Delta, \Omega), \quad b(\cdot, \cdot)=b_{1}(\cdot, \cdot), \\
& b_{0}(x, y)=b_{1}(u, \sigma, 0,0 ; v, \tau)=(u, \Delta \tau)_{\mathcal{T}}+(\sigma, \Delta v-\tau) \mathcal{T} \quad \text { with } x=(u, \sigma), y=(v, \tau), \\
& \hat{b}(\hat{x}, y)=b_{1}(0,0, \widehat{\boldsymbol{u}}, \widehat{\boldsymbol{\sigma}} ; v, \tau)=-\langle\widehat{\boldsymbol{u}}, \tau\rangle_{\mathcal{S}}-\langle\widehat{\boldsymbol{\sigma}}, v\rangle_{\mathcal{S}} \quad \text { with } \hat{x}=(\widehat{\boldsymbol{u}}, \widehat{\boldsymbol{\sigma}}), y=(v, \tau) .
\end{aligned}
$$

According to [1, Theorem 3.3] it suffices to show the two inf-sup properties

$$
\begin{aligned}
& \text { [1. Ass. 3.1]: } \sup _{0 \neq(v, \tau) \in H_{0}(\Delta, \Omega) \times H(\Delta, \Omega)} \frac{b_{1}(u, \sigma, 0,0 ; v, \tau)}{\|(v, \tau)\| \nu_{1}} \gtrsim\|u\|+\|\sigma\| \quad \forall u, \sigma \in L_{2}(\Omega), \\
& \begin{aligned}
\text { 1. (18)]: } \sup _{0 \neq(v, \tau) \in \mathcal{V}_{1}} \frac{\langle\widehat{\boldsymbol{u}}, \tau\rangle_{\mathcal{S}}+\langle\widehat{\boldsymbol{\sigma}}, v\rangle_{\mathcal{S}}}{\|(v, \tau)\| \mathcal{V}_{1}} \gtrsim\|\widehat{\boldsymbol{u}}\|_{\Delta, \mathcal{S}}+\|\widehat{\boldsymbol{\sigma}}\|_{\Delta, \mathcal{S}} \\
\forall(\widehat{\boldsymbol{u}}, \widehat{\boldsymbol{\sigma}}) \in \mathbf{H}_{00}^{\Delta}(\mathcal{S}) \times \mathbf{H}^{\Delta}(\mathcal{S}),
\end{aligned}
\end{aligned}
$$

and the identity

$$
H_{0}(\Delta, \Omega) \times H(\Delta, \Omega)=\left\{(v, \tau) \in \mathcal{V}_{1} ;\langle\widehat{\boldsymbol{u}}, \tau\rangle_{\mathcal{S}}+\langle\widehat{\boldsymbol{\sigma}}, v\rangle_{\mathcal{S}}=0 \forall(\widehat{\boldsymbol{u}}, \widehat{\boldsymbol{\sigma}}) \in \mathbf{H}_{00}^{\Delta}(\mathcal{S}) \times \mathbf{H}^{\Delta}(\mathcal{S})\right\}
$$

This identity is true by Proposition 2. Lemma 15 shows that 24 holds:

$$
\begin{aligned}
\left(\|u\|^{2}+\|\sigma\|^{2}\right)^{1 / 2} & =\sup _{0 \neq\left(g_{1}, g_{2}\right) \in L_{2}(\Omega) \times L_{2}(\Omega)} \frac{\left(u, g_{1}\right)+\left(\sigma, g_{2}\right)}{\left(\left\|g_{1}\right\|^{2}+\left\|g_{2}\right\|^{2}\right)^{1 / 2}} \\
& \lesssim \sup _{0 \neq(v, \tau) \in H_{0}^{2}(\Omega) \times H(\Delta, \Omega)} \frac{b_{1}(u, \sigma, 0,0 ; v, \tau)}{\left(\|v\|_{2}^{2}+\|\tau\|_{\Delta}^{2}\right)^{1 / 2}} \\
& \leq \sup _{0 \neq(v, \tau) \in H_{0}(\Delta, \Omega) \times H(\Delta, \Omega)} \frac{b_{1}(u, \sigma, 0,0 ; v, \tau)}{\|(v, \tau)\|_{1}} \quad \forall u, \sigma \in L_{2}(\Omega) .
\end{aligned}
$$

Finally, Proposition 3 shows that (25) is satisfied. This finishes the proof of (23), and of the theorem. 
Equivalence of (1) and (17). By construction of (17), any solution $u \in H_{0}^{2}(\Omega)$ of (1) provides a solution $\mathbf{u}:=\left(u, \Delta u, \operatorname{tr}^{\Delta}(u), \operatorname{tr}^{\Delta}(\Delta u)\right) \in \mathcal{U}_{1}$ of $(17)$. In fact, the regularity $u \in H_{0}(\Delta, \Omega)$, together with $f \in L_{2}(\Omega)$, is sufficient for this conclusion.

To see the other direction we use that $(17)$ is uniquely solvable. Its solution $\mathbf{u}=(u, \sigma, \widehat{\boldsymbol{u}}, \widehat{\boldsymbol{\sigma}})$ satisfies $u \in H_{0}(\Delta, \Omega)$ and solves $\Delta^{2} u=f$ in $\Omega$, as can be seen as follows. Selecting smooth test functions $v$ and $\tau$ with supports on individual elements, one obtains $\sigma=\Delta_{\mathcal{T}} u$ and $\Delta_{\mathcal{T}} \sigma=f$, first in the distributional sense and then in $L_{2}(\Omega)$ by the regularity $\sigma, f \in L_{2}(\Omega)$. Second, denoting as usual $\widehat{\boldsymbol{u}}=\left(\widehat{\boldsymbol{u}}_{T}\right)_{T}, \widehat{\boldsymbol{\sigma}}=\left(\widehat{\boldsymbol{\sigma}}_{T}\right)_{T}$, and using test functions $v, \tau \in \mathcal{D}(\bar{T})$ for $T \in \mathcal{T}$, one concludes that $\widehat{\boldsymbol{u}}_{T}=\operatorname{tr}_{T}^{\Delta}(u)$ and $\widehat{\boldsymbol{\sigma}}_{T}=\operatorname{tr}_{T}^{\Delta}(\sigma)$ for any $T \in \mathcal{T}$ so that $u \in H_{0}(\Delta, \Omega)$ and $\sigma \in H(\Delta, \Omega)$ by Proposition 2. Altogether, $u \in H_{0}(\Delta, \Omega)$ solves $\Delta^{2} u=f$. Since any such function $u$ leads to a solution of (17), as noted before, one concludes the stronger regularity $u \in H_{0}^{2}(\Omega)$ by uniqueness of (17). Therefore, $u \in H_{0}^{2}(\Omega)$ solves (1).

\subsection{Proof of Theorem 13 .}

The proof of Theorem 13 is analogous to the one of Theorem 11. The equivalence between (1) and (20) holds as before. To show the well-posedness of (20) we repeat the steps that show the wellposedness of (17) where we only have to replace the corresponding ingredients. Specifically, the injectivity of $B_{2}^{*}: \mathcal{V}_{2} \rightarrow \mathcal{U}_{2}^{\prime}$ is obtained by using Propositions 7 and 9 instead of Proposition 2 (i) to deduce the continuity $(v, \tau) \in H_{0}^{2}(\Omega) \times H(\Delta, \Omega)$ of $(v, \tau) \in \mathcal{V}_{2}$ satisfying $b_{2}(\mathbf{u} ; v, \tau)=0 \forall \mathbf{u} \in \mathcal{U}_{2}$. Then Lemma 15 shows that $(v, \tau)=0$.

The inf-sup condition for $B_{2}$, corresponding to (23), is shown by the same framework, based on the two inf-sup conditions

$$
\begin{aligned}
& \sup _{0 \neq(v, \tau) \in H_{0}^{2}(\Omega) \times H(\Delta, \Omega)} \frac{b_{2}(u, \sigma, 0,0 ; v, \tau)}{\|(v, \tau)\| \mathcal{V}_{2}} \gtrsim\|u\|+\|\sigma\| \quad \forall u, \sigma \in L_{2}(\Omega), \\
& \sup _{0 \neq(v, \tau) \in \mathcal{V}_{2}} \frac{\langle\widehat{\boldsymbol{u}}, \tau\rangle_{\mathcal{S}}+\langle\widehat{\boldsymbol{\sigma}}, v\rangle_{\mathcal{S}}}{\|(v, \tau)\|_{\mathcal{V}_{2}}} \gtrsim\|\widehat{\boldsymbol{u}}\|_{2, \mathcal{S}}+\|\widehat{\boldsymbol{\sigma}}\|_{\mathrm{dDiv}, \mathcal{S}} \quad \forall(\widehat{\boldsymbol{u}}, \widehat{\boldsymbol{\sigma}}) \in \mathbf{H}_{00}^{\Delta, 2}(\mathcal{S}) \times \mathbf{H}^{-3 / 2,-1 / 2}(\mathcal{S}),
\end{aligned}
$$

and the identity

$$
H_{0}^{2}(\Omega) \times H(\Delta, \Omega)=\left\{(v, \tau) \in \mathcal{V}_{2} ;-\langle\widehat{\boldsymbol{u}}, \tau\rangle_{\mathcal{S}}+\langle\widehat{\boldsymbol{\sigma}}, v\rangle_{\mathcal{S}}=0 \forall(\widehat{\boldsymbol{u}}, \widehat{\boldsymbol{\sigma}}) \in \mathbf{H}_{00}^{\Delta, 2}(\mathcal{S}) \times \mathbf{H}^{-3 / 2,-1 / 2}(\mathcal{S})\right\} .
$$

This identity is true by Propositions 7 and 9, and (27) holds as we have seen with (26). Finally, Propositions 5 and 8 show that (28) is satisfied.

\section{$7 \quad$ Numerical examples}

According to Theorems 12 and 14, any conforming subspaces $\mathcal{U}_{1, h} \subset \mathcal{U}_{1}$ and $\mathcal{U}_{2, h} \subset \mathcal{U}_{2}$ yield quasi-optimal approximations $\mathbf{u}_{1, h} \in \mathcal{U}_{1, h}$ and $\mathbf{u}_{2, h} \in \mathcal{U}_{2, h}$, respectively, of the solution(s) $\mathbf{u}_{1}=$ $\left(u, \Delta u, \operatorname{tr}^{\Delta}(u), \operatorname{tr}^{\Delta}(\Delta u)\right)$ and $\mathbf{u}_{2}=\left(u, \Delta u, \operatorname{tr}^{\Delta, 2}(u), \operatorname{tr}^{\mathrm{dDiv}}(\mathbb{D}(\Delta u))\right)$ to 17 and $(20)$, respectively. (In fact, $\mathbf{u}_{1}=\mathbf{u}_{2}$.) Here, $u \in H_{0}^{2}(\Omega)$ solves (1), and $\mathbf{u}_{1, h}$ and $\mathbf{u}_{2, h}$ are the solutions of (19) and (21), respectively. 
The construction of discrete subspaces of $\mathcal{U}_{1}$ and $\mathcal{U}_{2}$ and their approximation properties is ongoing research. In the case of the Kirchhoff-Love model we have presented a fully discrete analysis in [12]. Here, we only select some discrete spaces in an ad hoc fashion and present the corresponding convergence results without proving any convergence orders. Also, the construction of appropriate Fortin operators (needed to take the approximation of optimal test functions into account) is left open. Test functions are approximated by selecting identical meshes for ansatz and test spaces, and increasing polynomial degrees in the test spaces (see [12 for details).

Specifically, we consider the two-dimensional case $d=2$, and use regular triangular meshes $\mathcal{T}$ of shape-regular elements, with mesh parameter $h:=h_{\mathcal{T}}:=\max _{T \in \mathcal{T}} \operatorname{diam}(T)$. The DPG method provides a built-in error estimator, the residual norm $\eta:=\left\|B_{i}\left(\mathbf{u}_{i}-\mathbf{u}_{i, h}\right)\right\|_{\mathcal{V}_{i}^{\prime}}$. (We generically use $\eta$ and select $i=1$ or $i=2$ as needed.) By the product form of the test spaces, $\eta$ is composed of local element contributions $\eta^{2}=\sum_{T \in \mathcal{T}} \eta(T)^{2}$. For the case with singular solution we use these indicators to perform adaptive DPG schemes, based on newest-vertex-bisection and Dörfler marking with parameter of one half.

\subsection{Example with smooth solution}

We take $\Omega=(0,1)^{2}$ and use the manufactured solution $u(x, y)=x^{2}(1-x)^{2} y^{2}(1-y)^{2}$.

To compare the approximations given by the schemes $(19)$ and $(21)$, we use piecewise constant functions on uniform meshes for $u_{i, h}$ and $\sigma_{i, h}$, and traces of the reduced Hsieh-Clough-Tocher (HCT) functions for both $\widehat{\boldsymbol{u}}_{i, h}$ and $\widehat{\boldsymbol{\sigma}}_{i, h}(i=1,2)$. These HCT traces use piecewise cubic polynomials for (standard) traces on edges and piecewise linear polynomials for normal derivatives on edges, subject to the regularity of stemming from $H^{2}(\Omega)$-functions. For the reduced HCT elements we refer to [3], and the traces we use are described in [13].

Figure 1 presents the $L_{2}(\Omega)$ approximation errors for $u$ and $\sigma=\Delta u$ along with the corresponding residual $\eta$. The results for scheme $(19)$ are on the left and for (21) on the right. It appears that in both cases we have an asymptotical behavior of $\left\|u-u_{i, h}\right\| \simeq\left\|\sigma-\sigma_{i, h}\right\| \simeq \eta=\mathcal{O}(h)$. This is expected for lowest order approximations of a smooth function.

\subsection{Example with singular solution}

The next example is taken from [13. We consider the non-convex domain from Figure 2 with reentrant corner at $(0,0)$. The outer angle at this corner is $\frac{3}{4} \pi$. We take the manufactured solution

$$
u(r, \varphi)=r^{1+\alpha}(\cos ((\alpha+1) \varphi)+C \cos ((\alpha-1) \varphi))
$$

with polar coordinates $(r, \varphi)$ centered at the origin. It holds $\Delta^{2} u=0=: f$. For the boundary conditions we prescribe the values of $\left.u\right|_{\Gamma}$ and $\left.\nabla u\right|_{\Gamma}$. The parameters $\alpha$ and $C$ are chosen such that $u$ and its normal derivative vanish on the boundary edges that meet at the origin. Here, we have $\alpha \approx 0.673583432147380$ and $C \approx 1.234587795273723$. It holds $u \in H^{2+\alpha-\varepsilon}(\Omega)$ but $\Delta u \notin H^{1}(\Omega)$.

The numerical results for the two schemes 19 (on the left) and (21) (on the right) are shown in Figure 3. As before, we plot the $L_{2}(\Omega)$-errors for $u$ and $\sigma=\Delta u$ along with the corresponding 

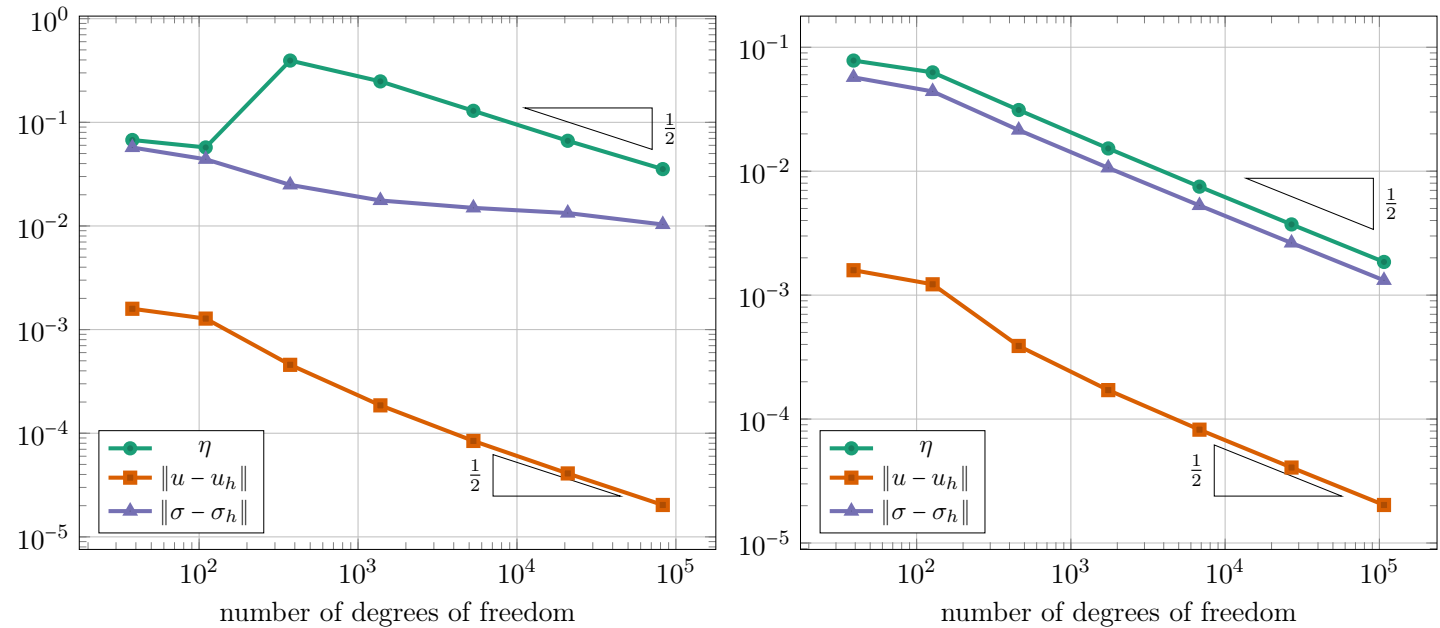

Figure 1: Errors generated by schemes (19) (left) and scheme (21) (right) for the smooth example from $\$ 7.1$.

residual $\eta$. In both cases the schemes converge at a low rate when using quasi-uniform meshes (curves without label "adap"), variant (19) being extremely slow. The rates exhibited by the second scheme are as expected by the regularity of $\sigma$. However, scheme 19 seems to suffer from the approximation of $\widehat{\boldsymbol{\sigma}}_{h}$ by smooth $H^{2}$-traces. This is clearly not an efficient basis. We can only claim convergence based on a density argument.

We have also used adaptive variants of both DPG schemes (curves with label "adap" in the same figures). It turns out that the second scheme (21) recovers its optimal rate of $\mathcal{O}\left(\operatorname{dim}\left(\mathcal{U}_{2, h}\right)^{-1 / 2}\right)$. On the other hand, the residual $\eta$ and error $\left\|\sigma-\sigma_{1, h}\right\|$ of the first scheme converge as slowly as before. Again, this seems to be caused by the inappropriate basis for $\widehat{\boldsymbol{\sigma}}_{1, h}$. It is an open problem to construct discrete trace spaces that improve the convergence rate of scheme 119 for non-smooth solutions. 


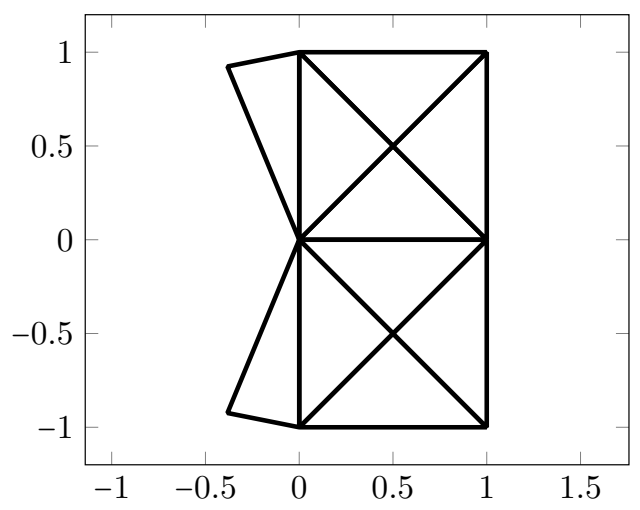

Figure 2: The non-convex domain with initial mesh.
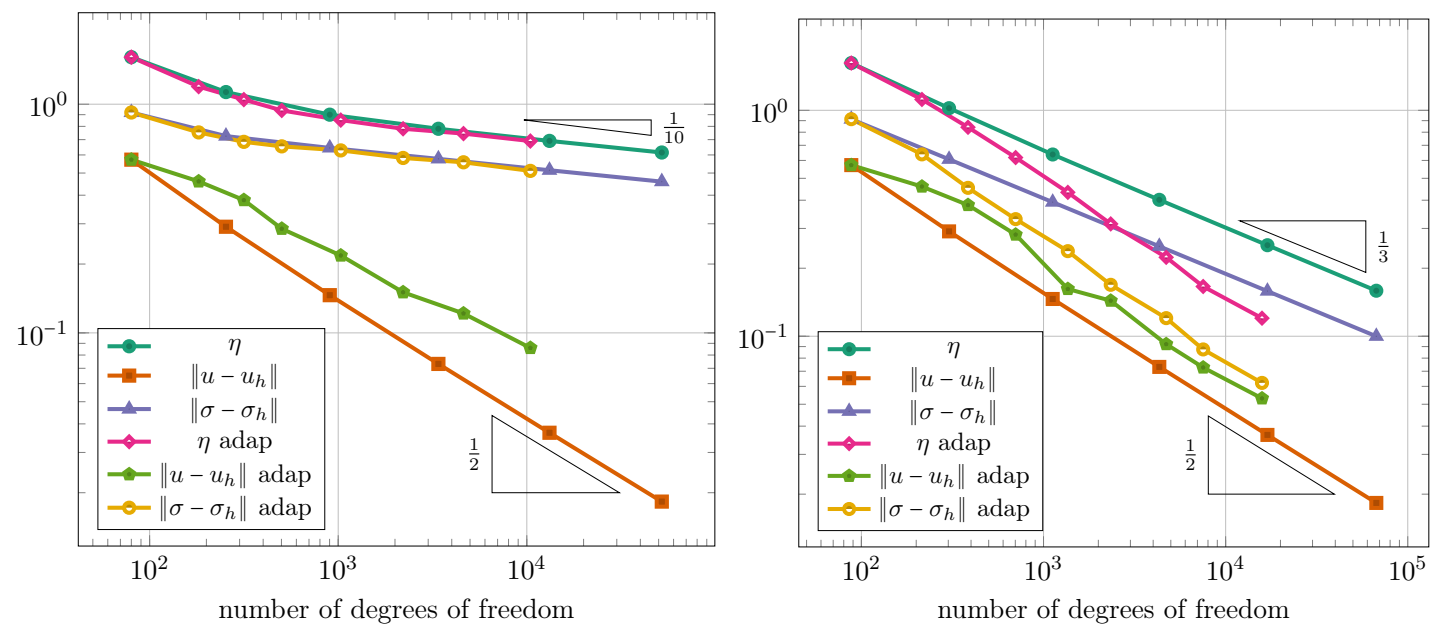

Figure 3: Errors generated by schemes (19) (left) and 21) (right) for the singular example from $\$ 7.2$. 


\section{A The space $\operatorname{tr}^{2, \Delta}(H(\Delta, \Omega))$ is not closed in $H^{2}(\mathcal{T})^{\prime}$.}

We give an example for the two-dimensional case and conjecture that the result is true in arbitrary space dimension $d \geq 2$, replacing the Dirac delta below by distributions supported on $(d-1)$ simplexes.

We consider a domain in $\mathbb{R}^{2}$ with a vertex, for instance the open triangle $\Omega=T$ with vertices $(0,0),(1,0),(0,1)$. The construction that follows also works for a Lipschitz domain where part of the boundary (connected, non-zero relative measure) is $C^{2}$, considering an interior point of this smooth part instead of a vertex.

Let us recall the definition of the trace operator $\operatorname{tr}_{T}^{2, \Delta}$, cf. (13),

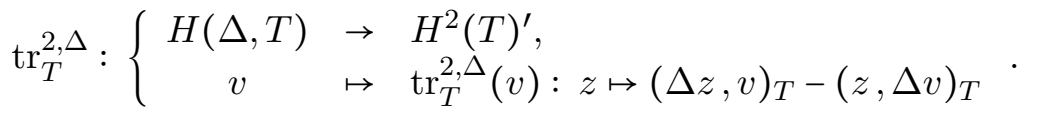

To show that $\operatorname{tr}_{T}^{2, \Delta}(H(\Delta, T))$ is not closed we construct a sequence of smooth functions $\left(v_{\varepsilon}\right)_{\varepsilon}$ (e.g., $\varepsilon=1 / n$ with positive integer $n$ ) so that the corresponding trace sequence $\left(\operatorname{tr}_{T}^{2, \Delta}\left(v_{\varepsilon}\right)\right)_{\varepsilon} \subset$ $\operatorname{tr}_{T}^{2, \Delta}(H(\Delta, T))$ converges in $H^{2}(T)^{\prime}$ to the Dirac distribution at $(0,0)$. This distribution is an element of the trace space $\mathbf{H}^{-3 / 2,-1 / 2}(\partial T)=\operatorname{tr}_{T}^{\mathrm{dDiv}}(H(\operatorname{div} \operatorname{div}, T))$, cf. [13], but it is not the trace of an $H(\Delta, T)$-function, cf. Lemma 10 and Appendix B.

We remark that this construction does not contradict the closedness of the trace space $\mathbf{H}^{\Delta}(\partial T)=\operatorname{tr}_{T}^{\Delta}(H(\Delta, T)) \subset H(\Delta, T)^{\prime}$ proved by Lemma 1 . Indeed, Dirac distributions at boundary points $e$ are not elements of $H(\Delta, T)^{\prime}$ since, e.g., $w=\log |\cdot, e|$ satisfies $w \in H(\Delta, T)$ (because $\Delta w=0)$, but its value at $e$ is not controlled.

We start by considering the mollifier type functions

$$
\phi_{\varepsilon}(t):= \begin{cases}C \frac{1}{\varepsilon} e^{-\varepsilon^{2} /\left(\varepsilon^{2}-t^{2}\right)}, & t \in[0, \varepsilon), \\ 0, & \text { else, }\end{cases}
$$

where $C>0$ is chosen such that $\int_{0}^{1} \phi_{\varepsilon}(t) d t=1 / 2$. Note that $C$ is independent of $\varepsilon$.

In the following let us denote $I=(0,1)$ and $I_{\varepsilon}=(0, \varepsilon)$. We need two technical results.

Lemma 16. We have that

$$
\left\|t \mapsto t \phi_{\varepsilon}(t)\right\|_{I} \rightarrow 0 \quad \text { as } \quad \varepsilon \rightarrow 0 .
$$

(Here and in the following, $\|\cdot\|_{I}$ denotes the $L_{2}(I)$-norm.)

Proof. Since $\phi_{\varepsilon}(t)$ takes its maximum at $t=0$ we can bound

$$
\left\|t \mapsto t \phi_{\varepsilon}(t)\right\|_{I}^{2} \leq C^{2} e^{-2} \frac{1}{\varepsilon^{2}} \int_{0}^{\varepsilon} t^{2} d t=\mathcal{O}(\varepsilon) .
$$


Lemma 17. Let $\varepsilon>0$ and $v \in H^{1}\left(I_{\varepsilon}\right)$ with $v(0)=0$. It holds the bound

$$
\|v\|_{L_{\infty}\left(I_{\varepsilon}\right)} \lesssim \varepsilon^{1 / 2}\left\|v^{\prime}\right\|_{I_{\varepsilon}}
$$

with hidden constant independent of $\varepsilon$ and $v$.

Proof. The statement follows in the standard way by using the continuous embedding $H^{1}(I) \hookrightarrow$ $C^{0}(\bar{I})$, a Poincaré inequality, and scaling arguments.

Now, using $\phi_{\varepsilon}$, we define $v_{\varepsilon} \in C^{\infty}(T)$ by

$$
v_{\varepsilon}(x, y):=-(x+y) \phi_{\varepsilon}(|(x, y)|)=-(x+y) \begin{cases}C \frac{1}{\varepsilon} e^{-\varepsilon^{2} /\left(\varepsilon^{2}-x^{2}-y^{2}\right)}, & |(x, y)|<\varepsilon, \\ 0, & \text { else. }\end{cases}
$$

Theorem 18. Let $\delta \in \mathbf{H}^{-3 / 2,-1 / 2}(\partial T) \subset H^{2}(T)^{\prime}$ denote the Dirac distribution supported at $(0,0)$, that is, $\langle\delta, z\rangle_{\partial T}=z(0,0)$ for $z \in H^{2}(T)$. It holds

$$
\operatorname{tr}_{T}^{2, \Delta}\left(v_{\varepsilon}\right) \rightarrow \delta \quad(\varepsilon \rightarrow 0) \quad \text { in } \quad H^{2}(T)^{\prime}
$$

Proof. Since $v_{\varepsilon}$ is smooth we can represent its trace as

$$
\left\langle\operatorname{tr}_{T}^{2, \Delta}\left(v_{\varepsilon}\right), z\right\rangle_{\partial T}=\left\langle\partial_{\mathbf{n}} z, v_{\varepsilon}\right\rangle_{\partial T}-\left\langle z, \partial_{\mathbf{n}} v_{\varepsilon}\right\rangle_{\partial T} \quad \forall z \in H^{2}(T) .
$$

To obtain a representation of $\partial_{\mathbf{n}} v_{\varepsilon}$ we note that for $\varepsilon<1 / 2, v_{\varepsilon}$ and its derivatives vanish on the edge spanned by the nodes $(1,0),(0,1)$. Second, we have that

$$
\nabla v_{\varepsilon}(x, y)=\frac{C}{\varepsilon} e^{-\varepsilon^{2} /\left(\varepsilon^{2}-x^{2}-y^{2}\right)}\left(\begin{array}{l}
-1 \\
-1
\end{array}\right)+\frac{C(x+y)}{\varepsilon} e^{-\varepsilon^{2} /\left(\varepsilon^{2}-x^{2}-y^{2}\right)} \frac{\varepsilon^{2}}{\left(\varepsilon^{2}-x^{2}-y^{2}\right)^{2}}\left(\begin{array}{l}
2 x \\
2 y
\end{array}\right) .
$$

Let $E:=(0,1) \times\{0\}$. Then, $\mathbf{n}_{E}=(0,-1)^{\top}$ and

$$
\left.\partial_{\mathbf{n}_{E}} v_{\varepsilon}\right|_{E}(t)=\mathbf{n}_{E} \cdot \nabla v_{\varepsilon}(t, 0)=\frac{C}{\varepsilon} e^{-\varepsilon^{2} /\left(\varepsilon^{2}-t^{2}\right)}=\phi_{\varepsilon}(t) .
$$

Here, $t=x$ is the (local) arc length of $E$ starting at $(0,0)$. Similarly, we calculate $\left.\partial_{\mathbf{n}_{E^{\prime}}} v_{\varepsilon}\right|_{E^{\prime}}(t)=$ $\phi_{\varepsilon}^{-}(t):=\phi_{\varepsilon}(1-t)$ where $t=1-y$ is the arc length of $E^{\prime}$ starting at $(0,1)$.

Now, for $z \in H^{2}(T)$, we find that

$$
\left\langle\operatorname{tr}_{T}^{2, \Delta}\left(v_{\varepsilon}\right), z\right\rangle_{\partial T}=\left\langle v_{\varepsilon}, \partial_{\mathbf{n}} z\right\rangle_{\partial T}-\left\langle\partial_{\mathbf{n}} v_{\varepsilon}, z\right\rangle_{\partial T}=\left\langle v_{\varepsilon}, \partial_{\mathbf{n}} z\right\rangle_{E}+\left\langle v_{\varepsilon}, \partial_{\mathbf{n}} z\right\rangle_{E^{\prime}}-\left\langle\phi_{\varepsilon}, z\right\rangle_{E}-\left\langle\phi_{\varepsilon}^{-}, z\right\rangle_{E^{\prime}}
$$

with $L_{2}(E)$-duality $\langle\cdot, \cdot\rangle_{E}$, and correspondingly for $E^{\prime}$. Note that

$$
\left\langle v_{\varepsilon}, \partial_{\mathbf{n}} z\right\rangle_{E} \leq\left\|v_{\varepsilon}\right\|_{E}\|\nabla z\|_{E} \lesssim\left\|v_{\varepsilon}\right\|_{E}\|z\|_{2, T}=\left\|t \mapsto t \phi_{\varepsilon}(t)\right\|_{I}\|z\|_{2, T} .
$$

We obtain the very same estimate replacing $E$ by $E^{\prime}$. Lemma 16 then proves that

$$
\sup _{0 \neq z \in H^{2}(T)} \frac{\left\langle v_{\varepsilon}, \partial_{\mathbf{n}} z\right\rangle}{\|z\|_{2, T}} \lesssim\left\|t \mapsto t \phi_{\varepsilon}(t)\right\|_{I} \rightarrow 0 \quad(\varepsilon \rightarrow 0) .
$$


To rewrite and estimate the term $\left\langle\partial_{\mathbf{n}} v_{\varepsilon}, z\right\rangle_{E}$ we use the representation (29) and the fact that $\int_{0}^{1} \phi_{\varepsilon}(t) d t=1 / 2$. This shows that

$$
\left\langle\partial_{\mathbf{n}} v_{\varepsilon}, z\right\rangle_{E}=\left\langle\phi_{\varepsilon}, z(\cdot, 0)\right\rangle_{I}=\frac{1}{2} z(0,0)+\left\langle\phi_{\varepsilon}, z(\cdot, 0)-z(0,0)\right\rangle_{I} \quad\left(z \in H^{2}(T)\right) .
$$

Analogously it holds

$$
\left\langle\partial_{\mathbf{n}} v_{\varepsilon}, z\right\rangle_{E^{\prime}}=\left\langle\phi_{\varepsilon}^{-}, z(0,1-\cdot)\right\rangle_{I}=\frac{1}{2} z(0,0)+\left\langle\phi_{\varepsilon}^{-}, z(0,1-\cdot)-z(0,0)\right\rangle_{I} \quad\left(z \in H^{2}(T)\right) .
$$

The last term in the latter two estimates can be estimated as follows (we only consider the first one). Since $\operatorname{supp}\left(\left.\phi_{\varepsilon}\right|_{I}\right)=[0, \varepsilon]$, Lemma 17 and a trace inequality show that

$$
\begin{aligned}
\left|\left\langle\phi_{\varepsilon}, z(\cdot, 0)-z(0,0)\right\rangle_{I}\right| & \leq\left\|\phi_{\varepsilon}\right\|_{L_{1}\left(I_{\varepsilon}\right)}\|z(\cdot, 0)-z(0,0)\|_{L_{\infty}\left(I_{\varepsilon}\right)} \\
& \lesssim \varepsilon^{1 / 2}\left\|\partial_{x} z\right\|_{I_{\varepsilon}} \leq \varepsilon^{1 / 2}\left\|\partial_{x} z\right\|_{I} \lesssim \varepsilon^{1 / 2}\|z\|_{2, T} .
\end{aligned}
$$

Now, using the delta distribution $\langle\delta, z\rangle_{\partial T}=z(0,0)$, we therefore obtain for any $z \in H^{2}(T)$

$$
\left|\langle\delta, z\rangle_{\partial T}-\left\langle\partial_{\mathbf{n}} v_{\varepsilon}, z\right\rangle_{\partial T}\right|=\left|\left\langle\phi_{\varepsilon}, z(\cdot, 0)-z(0,0)\right\rangle_{I}+\left\langle\phi_{\varepsilon}^{-}, z(0,1-\cdot)-z(0,0)\right\rangle_{I}\right| \lesssim \varepsilon^{1 / 2}\|z\|_{2, T} .
$$

This bound, together with (30), shows that

$$
\sup _{0 \neq z \in H^{2}(T)} \frac{\left\langle\delta-\operatorname{tr}_{T}^{2, \Delta}\left(v_{\varepsilon}\right), z\right\rangle_{\partial T}}{\|z\|_{2, T}} \leq \sup _{0 \neq z \in H^{2}(T)} \frac{\langle\delta, z\rangle_{\partial T}-\left\langle\partial_{\mathbf{n}} v_{\varepsilon}, z\right\rangle_{\partial T}}{\|z\|_{2, T}}+\sup _{0 \neq z \in H^{2}(T)} \frac{\left\langle v_{\varepsilon}, \partial_{\mathbf{n}} z\right\rangle_{\partial T}}{\|z\|_{2, T}} \rightarrow 0
$$

when $\varepsilon \rightarrow 0$. This finishes the proof.

\section{B The Dirac mass is not an element of $\operatorname{tr}^{2, \Delta}(H(\Delta, \Omega))$.}

The following argument is essentially the observation that fundamental solutions to the Laplacian (in any space dimension $\geq 2$ ) are not bounded. For illustration we show details for the case $d=2$. Without loss of generality we assume that $\Omega$ is the upper half of a circle with center $x_{0}=(0,0)$ and radius 1. (A smoothness of the boundary apart from Lipschitz continuity is not needed in our construction.) We define the points $x_{n}=\mathbf{n}\left(x_{0}\right) \frac{1}{n}=\left(0,-\frac{1}{n}\right) \notin \bar{\Omega}$ and consider the sequence

$$
\left(v_{n}\right)_{n} \quad \text { with } \quad v_{n}:=\log \left|x_{n}-\cdot\right| \in H^{2}(\Omega) .
$$

Since this sequence converges pointwise in $\Omega$ to $v:=\log |\cdot|$ and is bounded in $L_{2}(\Omega)$, it converges in $L_{2}(\Omega)$ to $v$. It also converges in $H(\Delta, \Omega)$ to $v$ because $\Delta v_{n}=\Delta v=0$ in $\Omega$.

Now we argue by contradiction. Suppose there exists $\sigma \in H(\Delta, \Omega)$ with $\operatorname{tr}_{\Omega}^{2, \Delta}(\sigma)=\delta_{x_{0}}$ (the Dirac delta supported at $\left.x_{0}\right)$, i.e., $\left\langle\operatorname{tr}_{\Omega}^{2, \Delta}(\sigma), z\right\rangle_{\Gamma}=z\left(x_{0}\right)$ for all $z \in H^{2}(\Omega)$.

Since $\sigma, v \in H(\Delta, \Omega)$ the value $\left\langle\operatorname{tr}_{\Omega}^{\Delta}(\sigma), v\right\rangle_{\Gamma}$ has to be finite. Moreover, since $v_{n} \rightarrow v$ in $H(\Delta, \Omega)$ as $n \rightarrow \infty,\left\langle\operatorname{tr}_{\Omega}^{\Delta}(\sigma), v_{n}\right\rangle_{\Gamma} \rightarrow\left\langle\operatorname{tr}_{\Omega}^{\Delta}(\sigma), v\right\rangle_{\Gamma}<\infty$. However, since $v_{n} \in H^{2}(\Omega)$ we conclude that

$$
\left\langle\operatorname{tr}_{\Omega}^{\Delta}(\sigma), v_{n}\right\rangle_{\Gamma}=\left\langle\operatorname{tr}_{\Omega}^{2, \Delta}(\sigma), v_{n}\right\rangle_{\Gamma}=v_{n}\left(x_{0}\right) \rightarrow \infty \text { as } n \rightarrow \infty .
$$

This contradicts $\left\langle\operatorname{tr}_{\Omega}^{\Delta}(\sigma), v\right\rangle_{\Gamma}<\infty$. 


\section{On the regularity of solutions to the bi-Laplace problem.}

We note a regularity result for the bi-Laplacian. Generally, a solution $u$ to problem (1) is a priorily sought in $H_{0}^{2}(\Omega)$. In this respect, $(12)$ is a fundamental relation to show the ellipticity (coercivity) of the induced bilinear form $(\Delta \cdot, \Delta \cdot)$. On the other hand, for a right-hand side function $f \in L_{2}(\Omega)$, the proof of Theorem 11 shows that this regularity is automatically satisfied.

Theorem 19. Let $u \in H_{0}(\Delta, \Omega)$ with $\Delta u \in H(\Delta, \Omega)$. It holds $u \in H_{0}^{2}(\Omega)$ so that, in particular, $\|\Delta u\|=\|\varepsilon \nabla u\|$.

Proof. By assumption, $u \in H_{0}(\Delta, \Omega)$ satisfies $f:=\Delta^{2} u \in L_{2}(\Omega)$. The proof of Theorem 11 shows that this problem has a unique solution $u \in H_{0}^{2}(\Omega)$. Then, $\|\Delta u\|=\|\varepsilon \nabla u\|$ holds by (12).

\section{References}

[1] C. Carstensen, L. F. Demkowicz, and J. Gopalakrishnan, Breaking spaces and forms for the DPG method and applications including Maxwell equations, Comput. Math. Appl., 72 (2016), pp. 494-522.

[2] P. G. Ciarlet, The Finite Element Method for Elliptic Problems, North-Holland, Amsterdam, 1978.

[3] P. G. Ciarlet, Interpolation error estimates for the reduced Hsieh-Clough-Tocher triangle, Math. Comp., 32 (1978), pp. 335-344.

[4] P. G. Ciarlet and P.-A. Raviart, A mixed finite element method for the biharmonic equation, Math. Res. Center, Univ. of Wisconsin-Madison, Academic Press, New York, 1974, pp. 125-145. Publication No. 33.

[5] B. Cockburn, B. Dong, And J. Guzmán, A hybridizable and superconvergent discontinuous Galerkin method for biharmonic problems, J. Sci. Comput., 40 (2009), pp. 141-187.

[6] M. Costabel and M. Dauge, Invertibility of the biharmonic single layer potential operator, Integral Equations Operator Theory, 24 (1996), pp. 46-67.

[7] C. De Coster, S. Nicaise, and G. Sweers, Solving the biharmonic Dirichlet problem on domains with corners, Math. Nachr., 288 (2015), pp. 854-871.

[8] L. F. Demkowicz and J. Gopalakrishnan, Analysis of the DPG method for the Poisson problem, SIAM J. Numer. Anal., 49 (2011), pp. 1788-1809.

[9] — A class of discontinuous Petrov-Galerkin methods. Part II: Optimal test functions, Numer. Methods Partial Differential Eq., 27 (2011), pp. 70-105.

[10] L. F. Demkowicz And N. Heuer, Robust DPG method for convection-dominated diffusion problems, SIAM J. Numer. Anal., 51 (2013), pp. 2514-2537. 
[11] T. FÜHRER, Superconvergent DPG methods for second-order elliptic problems, Comput. Methods Appl. Math. Appeared online, DOI:10.1515/cmam-2018-0250.

[12] T. Führer And N. Heuer, Fully discrete DPG methods for the Kirchhoff-Love plate bending model, Comput. Methods Appl. Mech. Engrg., 343 (2019), pp. 550-571.

[13] T. Führer, N. Heuer, And A. H. Niemi, An ultraweak formulation of the Kirchhoff-Love plate bending model and DPG approximation, Math. Comp., 88 (2019), pp. 1587-1619.

[14] T. Gerasimov, A. Stylianou, And G. Sweers, Corners give problems when decoupling fourth order equations into second order systems, SIAM J. Numer. Anal., 50 (2012), pp. 1604-1623.

[15] V. Girault And P.-A. Raviart, Finite element methods for Navier-Stokes equations, vol. 5 of Springer Series in Computational Mathematics, Springer-Verlag, Berlin, 1986. Theory and algorithms.

[16] R. Glowinski and O. Pironneau, Numerical methods for the first biharmonic equation and the two-dimensional Stokes problem, SIAM Rev., 21 (1979), pp. 167-212.

[17] P. Grisvard, Elliptic Problems in Nonsmooth Domains, Pitman Publishing Inc., Boston, 1985.

[18] N. Heuer and M. Karkulik, A robust DPG method for singularly perturbed reactiondiffusion problems, SIAM J. Numer. Anal., 55 (2017), pp. 1218-1242.

[19] J.-L. Lions And E. Magenes, Non-Homogeneous Boundary Value Problems and Applications I, Springer-Verlag, New York, 1972.

[20] P. B. Monk, An iterative finite element method for approximating the biharmonic equation, Math. Comp., 51 (1988), pp. 451-476.

[21] W. ZuleHner, The Ciarlet-Raviart method for biharmonic problems on general polygonal domains: mapping properties and preconditioning, SIAM J. Numer. Anal., 53 (2015), pp. $984-1004$. 